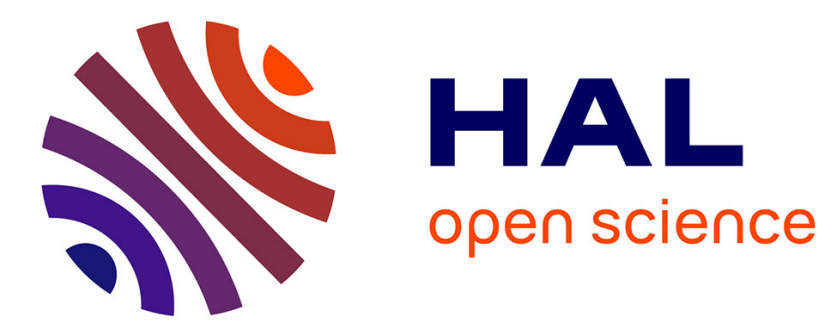

\title{
La dynamique de changement des formes de gouvernance: le cas français (2000-2014)
}

Xavier Hollandts, Daniela Borodak, Ariane Tichit

\section{To cite this version:}

Xavier Hollandts, Daniela Borodak, Ariane Tichit. La dynamique de changement des formes de gouvernance: le cas français (2000-2014). Finance Contrôle Stratégie, 2018. hal-02022915

\section{HAL Id: hal-02022915 https://hal.science/hal-02022915}

Submitted on 18 Feb 2019

HAL is a multi-disciplinary open access archive for the deposit and dissemination of scientific research documents, whether they are published or not. The documents may come from teaching and research institutions in France or abroad, or from public or private research centers.
L'archive ouverte pluridisciplinaire HAL, est destinée au dépôt et à la diffusion de documents scientifiques de niveau recherche, publiés ou non, émanant des établissements d'enseignement et de recherche français ou étrangers, des laboratoires publics ou privés. 


\section{Finance Contrôle Stratégie}

21-3 | 2018

Varia

\section{La dynamique de changement des formes de gouvernance : le cas français (2000-2014)}

Xavier Hollandts, Daniela Borodak et Ariane Tichit

\section{(2) OpenEdition}

Édition électronique

URL : http://journals.openedition.org/fcs/2965

ISSN : 2261-5512

Éditeur

Association FCS

Référence électronique

Xavier Hollandts, Daniela Borodak et Ariane Tichit, «La dynamique de changement des formes de gouvernance : le cas français (2000-2014) », Finance Contrôle Stratégie [En ligne], 21-3 | 2018, mis en ligne le 11 janvier 2019, consulté le 11 janvier 2019. URL : http://journals.openedition.org/fcs/2965

Ce document a été généré automatiquement le 11 janvier 2019.

Tous droits réservés 


\title{
La dynamique de changement des formes de gouvernance : le cas français (2000-2014)
}

\author{
Xavier Hollandts, Daniela Borodak et Ariane Tichit
}

Nous tenons sincèrement à remercier l'éditeur associé ainsi que les deux évaluateurs anonymes pour leurs suggestions et remarques très précieuses tout au long du processus de révision. Les seules erreurs éventuelles restantes relèvent de notre seule responsabilité.

\section{Introduction}

1 Pourquoi les entreprises changent-elles de structures de gouvernance ? Pourquoi passent-elles d'un conseil d'administration à une structure duale (directoire et conseil de surveillance) ? Pourquoi certaines entreprises ne changent-elles jamais de structure alors que d'autres réalisent des allers-retours incessants entre les différentes formes ${ }^{1}$ ? La littérature souligne que plusieurs facteurs peuvent conditionner ou expliquer le changement de structures de gouvernance. On peut penser à l'évolution du contexte réglementaire et aux évolutions de la « soft law» par exemple (Wirtz 2008). Bien évidemment, l'évolution de la structure de gouvernance est aussi la conséquence de facteurs endogènes à la gouvernance (Bonnet, Seville et Wirtz, 2017), impliquant les dirigeants, les administrateurs et dans une moindre mesure les actionnaires (Cannella, Jones et Withers 2015 ; Tihanyi, Graffin et George 2014). Si les transitions ponctuelles sont bien étudiées par la littérature (Joseph, Ocasio et Mc Donnell, 2014), en revanche la littérature est quasiment muette sur la dynamique de changements de structure de gouvernance (Marcel, Cowen et Ballinger 2017). Dans le cadre de cet article, nous nous focalisons non sur l'aspect "statique», mais sur la dynamique de changement des structures de gouvernance, en intégrant à la fois la dimension temporelle, mais également l'incidence des formes antérieures sur la propension des entreprises à conserver ou changer de structure de gouvernance. En cela, nous répondons à un appel de la communauté académique sur la nécessité d'analyser de façon dynamique la 
gouvernance des entreprises (Wintoki, Linck et Netter 2012). Notre article se focalise en particulier sur la stabilité et la durée des formes de gouvernance adoptées par les sociétés cotées françaises.

2 La question d'une gouvernance plus ou moins stable (dans le temps) est de première importance car elle affecte globalement son efficacité (Krause 2017). Depuis le rapport Cadbury (1992), l'attention des praticiens et des chercheurs s'est presque uniquement concentrée sur la forme de la gouvernance, sa composition et son fonctionnement (Boyd, Gove \& Solarino 2017 ; Joseph, Ocasio \& Mc Donnel 2014 ; Dalton \& Dalton 2011). Cependant, un paramètre majeur de l'efficacité de la gouvernance n'a pas pour l'heure été précisément étudié (Marcel et al. 2017). Dans cet article, nous considérons que la stabilité et la durabilité de la structure de gouvernance constituent un élément essentiel, nécessaire à la régulation des conflits d'agence potentiels (Kole \& Lehn 1999, 1997). Nous envisageons la stabilité de la gouvernance d'une entreprise comme sa persistance dans le temps, autrement dit l'absence de changement de forme de gouvernance dans le temps (Allen et Wood 2006) ${ }^{2}$. Enfin, la durabilité renvoie à la dimension temporelle, autrement dit la persistance dans un état (mesurée par sa durée en années ou en mois par exemple).

3 La stabilité de la gouvernance des entreprises, à l'instar de ce qui est observé dans les entreprises familiales par exemple (Charlier et Lambert 2013), est susceptible d'avoir une influence sur son efficacité globale. Une gouvernance stable, qui ne change ni de forme, ni de fonctionnement (voire même de composition), peut permettre l'exercice plus aisé du devoir de contrôle ou de surveillance des administrateurs (Marcel et al. 2017 ; Joseph et al. 2014 ; Kole \& Lehn 1999, 1997) comme cela peut éventuellement faciliter l'enracinement des dirigeants, qui ont toute latitude pour développer dans le temps une stratégie d'enracinement voire de " domination» du conseil et de ses membres (Krause 2017 ; Joseph et al. 2014 ; Fracassi et Tate 2012). Par ailleurs, l'instabilité de la gouvernance, qui se traduit par de fréquents et nombreux changements de structure peut également faciliter l'enracinement des dirigeants (Belot, Ginglinger, Slovin \& Sushka 2014). De nombreux changements de structures peuvent être accompagnés d'un changement de composition du conseil et de ses règles de fonctionnement, limitant en cela l'exercice du devoir de surveillance. Nous considérons que le processus aboutissant in fine à faire évoluer (ou non) la structure de gouvernance est le résultat d'une négociation impliquant les parties essentielles de la gouvernance (dirigeants, actionnaires, administrateurs - Boone, Casares Field, Karpoff et Raheja 2007). Dans cet article, nous entendons ainsi examiner l'influence de différentes variables sur la stabilité de la structure de gouvernance des entreprises cotées françaises : performance, structure de l'actionnariat, enracinement du dirigeant (Boone, et al. 2007; Hermalin et Weisbach 2003).

4 Les apports de notre article se situent à plusieurs niveaux. Nous contribuons à la littérature financière sur la gouvernance en mettant en relief l'importance de la dimension temporelle et dynamique de la structure de gouvernance. Pour cela, nous envisageons les changements de structures de gouvernance comme un processus dynamique de longue période, répondant ainsi à un appel de la communauté sur ce sujet (Boyd et al. 2017 ; Wintoki, Linck \& Netter 2012). En effet, la majorité des articles empiriques considèrent seulement la dernière structure de gouvernance observée dans les données disponibles, sans tenir compte de l'historique de l'entreprise en la matière. Notre contribution est aussi d'ordre méthodologique puisque nous mobilisons (pour la première fois à notre connaissance) des modèles de durée multi-états de type semi- 
markovien ${ }^{3}$ qui permettent précisément d'analyser les changements de structures de gouvernance, mais également leur durée dans le temps ${ }^{4}$. Ces modèles sont particulièrement adaptés à nos données. En effet, l'observation des entreprises françaises cotées sur la période 2000-2014 montre que ces entreprises connaissent plusieurs trajectoires, en adoptant des formes de gouvernance de durées différentes (graphique 1 ci-après).

5 Le cas français est très intéressant à étudier, car ce pays est le seul, depuis 1966, à avoir des entreprises qui peuvent choisir entre les deux structures classiques de gouvernance, conseil d'administration ou structure duale (directoire et conseil de surveillance). Par ailleurs, les entreprises françaises ont toujours la possibilité de changer de structure de gouvernance au cours de leur existence, ce qui fait de la France, un pays unique pour observer la stabilité des structures de gouvernance dans le temps (Belot et al. 2014). Le reste de l'article est organisé comme suit. Dans une première partie, nous décrivons brièvement le phénomène d'un point de vue statistique et nous analysons les facteurs ayant un impact sur la stabilité et la durée de la structure de gouvernance. Dans une seconde partie, nous décrivons la méthodologie de recherche et présentons les résultats obtenus sur notre échantillon d'entreprises françaises cotées. Dans une troisième partie, nous discutons des implications théoriques, empiriques et managériales des résultats de notre recherche.

\section{Section 1 : la dynamique de l'évolution des structures de gouvernance des sociétés cotées françaises}

\subsection{L'évolution des structures de gouvernance : un aperçu statistique}

6 Les entreprises cotées françaises ont la possibilité d'opter pour trois modalités de gouvernance distinctes. Les deux premières correspondent à la forme dite moniste (conseil d'administration) qui comporte deux modalités : (1) conseil d'administration avec P-DG (une même personne préside le conseil et exerce la fonction de directeur général) et (2) conseil d'administration avec dissociation des fonctions de président du conseil et de directeur général. Enfin, la troisième modalité correspond à la structure duale (3) composée d'un directoire associé à un conseil de surveillance. Les différents codes de gouvernance, comme la littérature académique, tendent à considérer que la forme " $\mathrm{CA}$ et PDG» (modalité 1) permet plus aisément au PDG d'exercer son influence et de " dominer» ainsi le conseil (voir Krause 2017 pour une synthèse). En revanche, la séparation des fonctions de P-DG et encore plus la structure duale représentent des formes plus autonomes vis-à-vis de l'influence du dirigeant exécutif (Boyd et al. 2017; Hollandts, Borodak et Tichit 2015). Ainsi, le choix de la structure de gouvernance conditionne pour partie l'efficacité potentielle de la fonction de surveillance traditionnellement dévolue au conseil (d'administration ou de surveillance). Cependant, observer seulement la dernière forme connue de gouvernance ne permet pas de saisir la dynamique à l'œuvre (Marcel et al. 2017). En effet, deux entreprises fonctionnant, pour une année donnée, avec une structure duale peuvent cacher deux trajectoires très différentes. La première peut toujours avoir fonctionné sous ce régime tandis que l'autre peut avoir basculé en structure duale depuis l'année précédente, après différentes " transitions $»^{5}$ potentielles. Notre perspective est dès lors d'analyser l'évolution et la 
dynamique des structures de gouvernance des entreprises cotées françaises sur une période relativement longue. Certaines entreprises connaissent ainsi une gouvernance très stable alors que d'autres changent régulièrement ou de manière " erratique » de forme de gouvernance. A titre d'illustration, nous avons sélectionné six entreprises affichant des comportements distincts (voir graphique 1 ci-après - l'ensemble des trajectoires sont reportées en annexe 2). Notre objectif est donc d'observer et d'analyser le processus dynamique ayant une influence sur la stabilité ou non de la gouvernance des entreprises. En cela, nous répondons à l'appel de la communauté qui souligne la nécessité de s'intéresser à la dimension dynamique de la gouvernance des entreprises, essentielle, mais peu documentée jusqu'à présent (Boyd et al. 2017 ; Tihanyi, Graffin et George 2014 ; Filatotchev, Toms et Wright 2006).

Graphique 1. Les trajectoires des formes de gouvernance de 6 entreprises cotées françaises 2000-2014

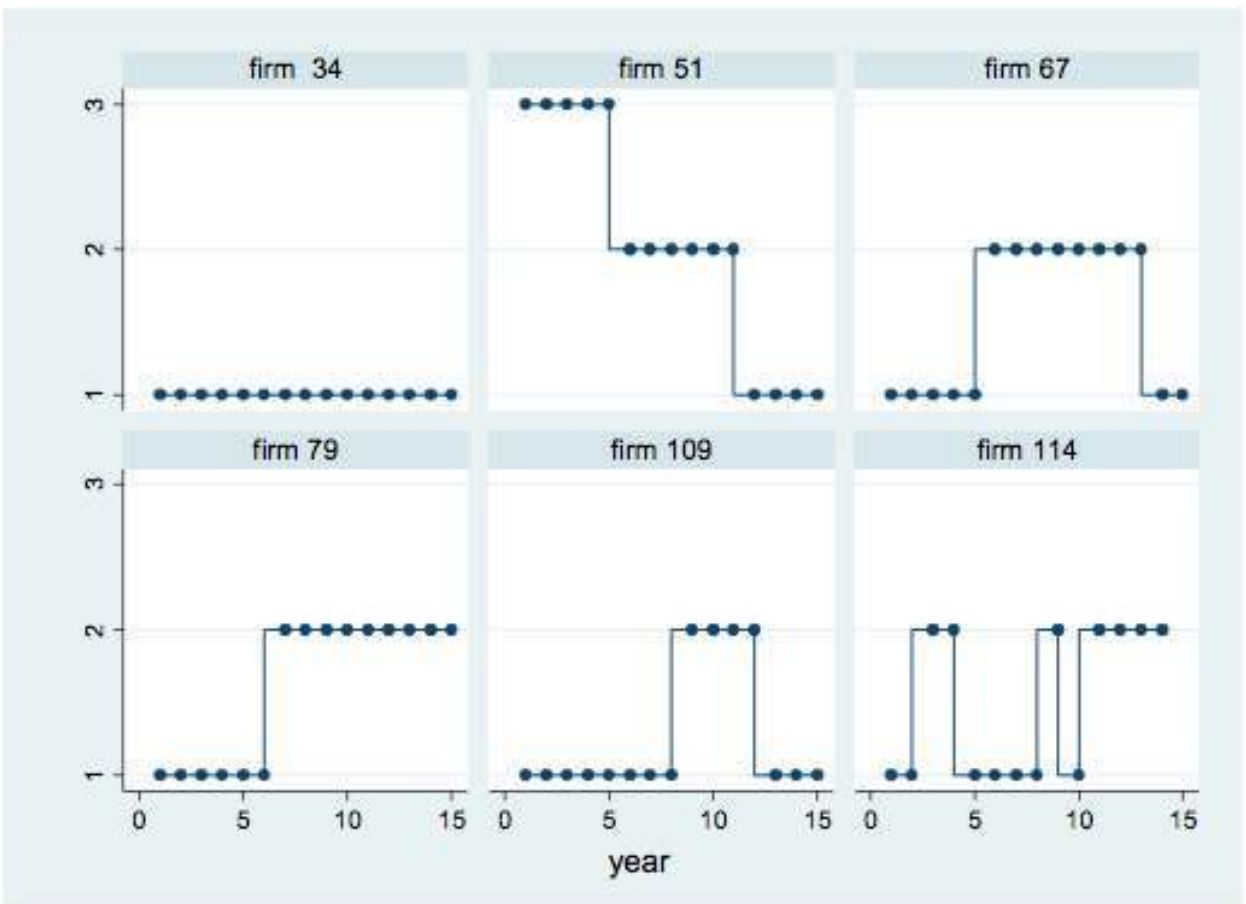

$7 \quad$ L'axe des ordonnées représente les trois formes de gouvernance possibles (cf. note de bas de page 1) et l'axe des abscisses représente la durée (en années). L'entreprise 34 ne change jamais de forme de gouvernance au cours de la période observée (elle a toujours connu la forme 1 - CA avec PDG pendant les 15 années d'observation). L'entreprise 79 change une fois de forme de gouvernance (elle évolue vers la forme " $\mathrm{CA}$ avec dissociation des fonctions de président/DG »). Les entreprises 51, 67 et 109 changent 3 fois de forme de gouvernance, à cela près que les deux dernières (67 et 109) reviennent vers leur forme de gouvernance initiale. L'entreprise 51 connait les 3 modalités sur la période d'observation, partant de la forme « structure duale » pour aboutir vers la forme « $\mathrm{CA}$ avec PDG » après une période transitoire de 6 années sous la forme " CA avec P-DG » (CA avec dissociation des fonctions de président/DG). L'entreprise 114 connait des allersretours répétés entre deux formes de gouvernance (elle totalise 5 transitions en 15 années d'observation). 
8 Ainsi, un simple aperçu de quelques entreprises issues du notre échantillon ${ }^{6}$ montre que la dynamique de changement de la gouvernance des entreprises cotées françaises peut être radicalement différente. D'un côté, nous pouvons observer des entreprises connaissant une grande stabilité dans le temps et ne changeant jamais de structure de gouvernance lors de la période d'observation. De l'autre, nous observons un certain nombre de changements, faibles ou nombreux, avec des durées variables. L'entreprise 79 reste 6 ans sous la forme « CA avec PDG » avant de passer 9 ans dans la forme « CA avec séparation P-DG »; l'entreprise 114 ne passe pas plus de 4 dans la forme « CA avec PDG» et pas plus de 2 ans dans la forme "CA avec séparation P-DG». Autre trajectoire intéressante, l'entreprise 51 réalise une transition continue de la forme duale (la plus « autonome ») vers la forme "CA avec PDG». Dernier élément, certaines entreprises connaissent des transitions progressives (entreprise 51) alors que d'autres multiplient les allers-retours (entreprises 109 et 114). Les différents types de transitions (changement de forme de gouvernance, notée ci-après FG) sont synthétisés dans le tableau 1 ci-dessous.

Tableau 1. Dynamique des changements de la forme de gouvernance

\begin{tabular}{|l|l|l|l|}
\hline Transition & & Effectif & Pourcentage \\
\hline FG1->FG1 & Pas de transition & 34 & $29 \%$ \\
\hline FG1->FG2 & Transition 1 & 38 & $32 \%$ \\
\hline FG1->FG3 & Transition 2 & 9 & $8 \%$ \\
\hline FG2->FG2 & Pas de transition & 3 & $3 \%$ \\
\hline FG2->FG1 & Transition 3 & 22 & $19 \%$ \\
\hline FG2->FG3 & Transition 4 & 0 & $0 \%$ \\
\hline FG3->FG3 & Pas de transition & 19 & $16 \%$ \\
\hline FG3->FG1 & Transition 5 & 17 & $14 \%$ \\
\hline FG3->FG2 & Transition 6 & 7 & $6 \%$ \\
\hline
\end{tabular}

Note :FG1 - CA avec PDG ; FG2 - CA avec séparation des fonctions ; FG3 - Directoire et conseil de surveillance

Les transitions les plus fréquentes concernent la forme « conseil d'administration» (la plus répandue parmi les sociétés cotées françaises) et en particulier dans le cas de la transition potentielle entre « CA avec PDG » - transition 1 dans le tableau ci-dessus - et " CA avec P-DG » (séparation des fonctions de président) - transition 3 dans le tableau cidessus. À elles seules, ces deux transitions concentrent $51 \%$ de l'ensemble des transitions (32\%+19\%). 


\subsection{Quelle dynamique d'évolution des structures de gouvernance?}

10 Les changements de structures de gouvernance sont le résultat d'un processus de négociation entre les parties prenantes essentielles de la gouvernance de l'entreprise (Belot et al. 2014, Boone et al. 2007 ; Hermalin et Weisbach 2003). Les dirigeants de l'entreprise, les administrateurs et les actionnaires sont concernés au premier chef. Audelà de ces individus centraux dans le processus de décision, le contexte environnant l'entreprise est également déterminant. En effet, une entreprise se trouvant en difficulté ou confrontée à un environnement concurrentiel compliqué peut être incitée à faire évoluer sa direction et/ou sa structure de gouvernance. Par ailleurs, des entreprises peuvent être incitées par "mimétisme» sectoriel à adopter les structures de gouvernance en vogue dans le secteur concerné, comme cela a pu être le cas dans l'industrie bancaire suite à la crise financière de 2008-2009 (Grove, Patelli, Victoravich, \& Xu 2011).

11 Si on examine la littérature sur le sujet, on relève ainsi deux grandes catégories de variables susceptibles d'influencer la dynamique de changement de forme de gouvernance. D'une part, des facteurs propres à la gouvernance (dirigeant, conseil, actionnariat) et d'autre part des facteurs liés au contexte de performance de l'entreprise (performance économique et secteur d'activité).

12 Concernant le dirigeant, la littérature académique souligne l'importance majeure des stratégies d'enracinement des dirigeants sur la dynamique de changement de gouvernance (Kole et Lehn 1999). La relation d'agence essentielle qui se noue entre les dirigeants, les actionnaires et les administrateurs pouvant être potentiellement plus ou moins conflictuelle, les dirigeants sont susceptibles de déployer des stratégies d'enracinement qui nuisent en général à l'efficacité globale de la gouvernance de l'entreprise (Boyd et al. 2011). En voulant diminuer l'efficacité du contrôle des administrateurs ou en voulant réduire le contrôle exercé par les actionnaires, les dirigeants sont susceptibles de déployer différents stratagèmes leur permettant ainsi de " contrôler " voire " dominer " dans une certaine mesure la structure de gouvernance (Krause 2017). Ces stratégies d'enracinement visent bien évidemment à assurer le maintien en poste du dirigeant concerné tout comme elles peuvent permettre de lui assurer l'extraction future de bénéfices privés (Mard, Marsat et Roux 2014). La littérature souligne que trois dimensions essentielles permettent d'approximer l'enracinement ou la propension à l'enracinement des dirigeants (Linck et al. 2008). La durée en poste du dirigeant est un élément essentiel et une plus grande longévité permet aux dirigeants de mieux résister aux différentes pressions internes comme externes (Boone et al. 2007; Hermalin et Weisbach, 2003). Deuxième élément, le taux de rotation mesurant le départ des dirigeants est également un indicateur de l'effectivité ou non des stratégies d'enracinement (Marcel et al. 2017). Enfin, la littérature relève que l'âge du dirigeant est un facteur essentiel pouvant expliquer les transitions potentielles et les changements de structures de gouvernance (Goyal et Park 2002). La proximité, notamment de la retraite, joue un double rôle (Belot et al. 2014). D'une part, cela modifie les stratégies d'enracinement des dirigeants qui sont toutes choses égales par ailleurs, relativement moins incités à mettre en œuvre des stratégies d'enracinement conduisant à ne surtout pas changer de forme de gouvernance (Hollandts et al. 2015). En effet, un PDG ou un DG est de plus en plus en mesure de dominer son conseil à mesure que la structure de 
gouvernance n'évolue pas. Il est alors susceptible de déployer des routines et des connivences avec les membres du conseil (Joseph et al. 2014). Cela est surtout observé dans le cas des conseils d'administration et notamment dans les situations où le DG préside également le conseil (Krause 2017 ; Fracassi \& Tate 2012). Ainsi, l'évolution de la gouvernance vers la séparation des fonctions ou la forme duale est moins intéressante pour un DG souhaitant s'enraciner ou souhaitant disposer de bénéfices privés. Il/elle a donc tout intérêt à ne pas faire évoluer la gouvernance de la société. Cependant, la proximité de la retraite du dirigeant peut inciter les autres parties prenantes (administrateurs, actionnaires) à faire évoluer la structure de gouvernance afin d'assurer une transition à la tête de l'entreprise. Il n'est en effet pas rare de séparer les fonctions de DG et de président, le temps que l'ancien DG assure la transition avec le nouveau DG. Cela est encore plus manifeste lors de la mise en place de structure duale, l'ancien DG glissant au conseil de surveillance et le nouveau DG assumant ses fonctions au sein du directoire (par exemple la récente transition observée chez Publicis - Maurice Levy devenant président du Conseil de surveillance et Arthur Sadoun présidant le Directoire ${ }^{7}$ ). Ainsi, de par son rôle ambivalent, l'âge du dirigeant et la proximité de la retraite sont susceptibles de jouer un rôle majeur dans les transitions (Belot et al. 2014; Goyal et Park 2002). Concernant la composition du conseil, il apparaît que notre période d'observation englobe un évènement majeur relatif à la mise en application de la loi Copé/Zimmerman concernant la féminisation des conseils. La littérature souligne que l'augmentation de la diversité de genre au sein des conseils est susceptible d'amener la gouvernance à évoluer y compris en changeant de forme voire de fonctionnement. Par ailleurs, la mise en œuvre de cette loi a souvent incité les entreprises à modifier leurs structures de gouvernance afin soit d'élargir la taille globale de leur conseil, soit en renouvelant leur conseil concomitamment à un changement de forme de gouvernance (Allemand et Brullebaut 2014 ; Gregoir, Maury \& Palomino 2013).

13 Au-delà de cette négociation interne, la structure actionnariale peut avoir une influence sur la stabilité de la structure de gouvernance (Sur et al. 2013 ; Kole et Lehn 1997). En raison de la relation d'agence consécutive à la séparation propriété/contrôle (Shleifer et Vishny 1997), les actionnaires peuvent être incités à faire évoluer les structures de gouvernance afin de s'assurer un degré de contrôle plus important. Cela est en revanche beaucoup moins intéressant pour des actionnaires concentrant une part, voire majoritaire, du capital. Par conséquent, nous supposons que la concentration du capital a un effet sur le potentiel de changement de la structure de gouvernance (Daily, Dalton et Rajagopalan 2003). Plus précisément, nous estimons que plus le capital est concentré et plus la probabilité d'évolution de la structure de gouvernance sera faible. En effet, ces actionnaires possèdent un pouvoir d'influence déterminant lié à la détention significative de droits de vote et à leur capacité à maitriser les assemblées générales d'actionnaires (Belot et al. 2014).

Deuxième série de variables, le contexte de performance caractérisant l'entreprise. Ces facteurs dits de "contingence» (l'environnement sectoriel et la performance de l'entreprise) sont susceptibles d'affecter la stabilité de la structure de gouvernance (Boyd et al. 2011 ; Bebchuck et Weisbach 2010). La littérature souligne que de mauvaises performances en soi ou relativement à celles des concurrents appartenant au même secteur d'activité peuvent inciter les acteurs de la gouvernance à faire évoluer la structure, raccourcissant de fait la durée de la structure de gouvernance en place (Aguilera, Filatotchev, Gospel \& Jackson 2008). La performance est donc un élément 
majeur pouvant conduire l'entreprise à faire évoluer plus ou moins rapidement sa structure de gouvernance si elle est jugée peu pertinente ou adéquate au regard des enjeux stratégiques et financiers affrontés par l'entreprise. Ceci est particulièrement sensible en cas de mauvaises performances et peut constituer un signal positif envoyé aux évaluateurs et aux marchés financiers (Belot et al. 2014 ; Grove et al. 2011). La mauvaise performance est donc susceptible d'inciter, toutes choses égales par ailleurs, les entreprises à faire évoluer plus rapidement la structure de gouvernance afin d'assurer un meilleur contrôle du comportement et des résultats du dirigeant (Raheja 2005). Ainsi le contexte de performance et l'environnement de l'entreprise constituent des facteurs de contingence susceptibles d'affecter la stabilité de la structure de gouvernance (et leur durée). La décision finale éventuelle de faire évoluer la structure de gouvernance revient aux parties prenantes directes de la gouvernance que sont les dirigeants, les administrateurs et les actionnaires. Le changement de structure résulte alors d'un jeu d'influence, de négociations et d'éventuels rapports de force entre ces différents acteurs (Belot et al. 2014, Boone et al. 2007 ; Hermalin et Weisbach 2003). Nous résumons cidessous les différents liens attendus.

Schéma 1. Modèle théorique et liens attendus

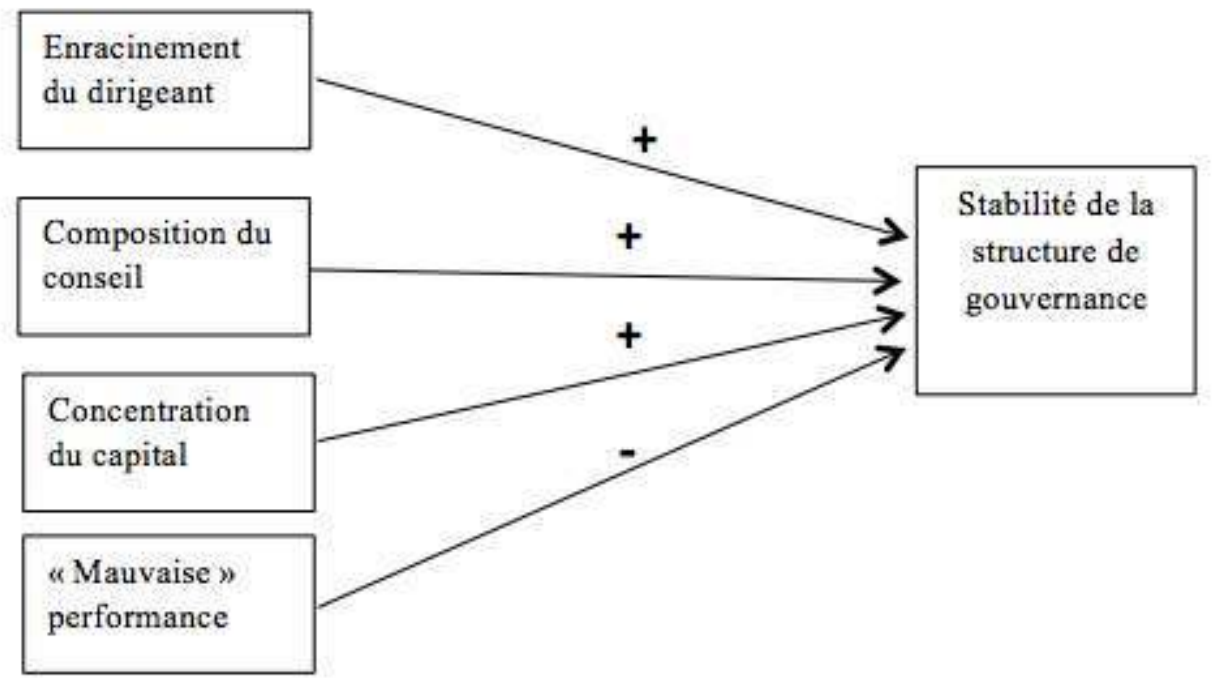

\section{Méthodologie de la recherche}

\subsection{Démarche économétrique}

La description de l'évolution des phénomènes que nous souhaitons analyser (les transitions et la durée des formes de gouvernance) comporte quatre particularités (Allison, 1982). La première tient à la troncature ${ }^{8}$ des données sur les phénomènes observés. Des données sont tronquées lorsqu'un évènement n'a pas pris fin lors de la dernière année d'observation de "l'individu statistique » (ici l'entreprise). Dans notre cas, nous pouvons considérer que toutes les données sont tronquées pour la dernière année d'observation (2014), car nous observons les entreprises dans une certaine forme de gouvernance sans savoir combien de temps elles vont y rester par la suite (en 2015 et après). Du fait de la présence de données tronquées, les modèles de durée sont les plus 
adéquats. La deuxième particularité tient au fait d'observer plusieurs transitions possibles pour une même société : en effet, les entreprises françaises cotées peuvent choisir entre trois modes différents de gouvernance et peuvent, à chaque période, changer pour une des deux autres formes. Il n'y a donc pas un seul chemin transitionnel possible, comme dans les modèles de durée simples, mais deux, à chaque point d'observation. Ceci impose dès lors l'utilisation de modèles de durée dits " multi-états ». La troisième complication tient au fait que certaines variables influençant potentiellement les choix de mode de gouvernance et leur durée sont variables dans le temps. Le temps joue en effet un rôle important et a une influence sur la forme observée à une période précise. Pour être plus précis, le temps passé dans une forme peut par exemple renforcer la probabilité de rester dans cette même forme l'année d'après. Ainsi, notre variable dépendante intègre à la fois une durée dans un état (une forme de gouvernance), la prise en compte de la nature de la transition, et le fait que la transition ait lieu ou non'. Cela implique d'estimer des modèles de durée multi-états en panel tout en intégrant l'influence des facteurs constants et variables pour chacune des entreprises observées. Enfin, la quatrième et dernière caractéristique est que les entreprises connaissent des évènements récurrents (états réversibles), c'est-à-dire que la même forme de gouvernance peut être observée plusieurs fois sur une même entreprise en des temps différents ${ }^{10}$ : dans ce cas, les phénomènes ne peuvent pas être considérés comme indépendants comme c'est le cas dans l'analyse de régression usuelle. Il est dès lors nécessaire de faire des hypothèses sur l'histoire de "l'individu statistique " (l'entreprise). L'ensemble de ces caractéristiques nous a conduits à utiliser les modèles multi-états en panel afin d'avoir une analyse plus fine des facteurs susceptibles d'influencer la dynamique complexe des changements de structure de gouvernance. Ces modèles ont été développés depuis une trentaine d'années et sont largement utilisés dans les études biomédicales (Conlon et al, 2014 ; Gauzère et al., 1999), ainsi qu'en démographie (Eulenburg et al., 2015 ; Farewell et Tom, 2014), économie et finance (Wiberg et al, 2017 ; Koopman et al., 2008 ; Jarrow et Turnbull, 1995), et dans les sciences politiques (Johnson, 2014).

16 Nous adoptons ici la démarche suggérée par Crowther et Lambert $(2016,2014)$ pour estimer des durées de persistance dans certains états lorsque plusieurs épisodes sont observés par entreprise. Celle-ci consiste à mettre en forme la base de données pour une analyse de type risques concurrents, et d'y appliquer des modèles de durée. Nous utilisons une méthode d'estimation qui consiste à modéliser la distribution du temps de séjour dans l'état par une fonction (Foucher et al. 2004, Perez-Ocon et Ruiz-Castro 1999), qui permet de choisir une distribution de durées et un nombre spécifique de variables pour chaque transition. Nous définissons dans un premier temps tous les états et toutes les transitions possibles d'un état à un autre. L'évolution dans le temps des formes de gouvernance est décrite par un schéma de Markov ${ }^{11}$ (voir schéma 1, ci-après, pour une présentation détaillée se reporter à l'annexe 3) : tous les ans, les entreprises restent soit dans le même état (FG inchangé), soit elles évoluent vers un état tout autre, avec une probabilité de transition d'un état à l'autre qui varie en fonction du temps. Les trois « états» discrets, mutuellement exclusifs et collectivement exhaustifs sont: FG1-si l'entreprise adopte la forme « conseil d'administration avec PDG »; FG2 - si elle adopte la forme " conseil d'administration avec séparation des fonctions de Président du conseil et de Directeur général »; FG3-si elle opte pour la forme duale « conseil de surveillance et directoire ». 


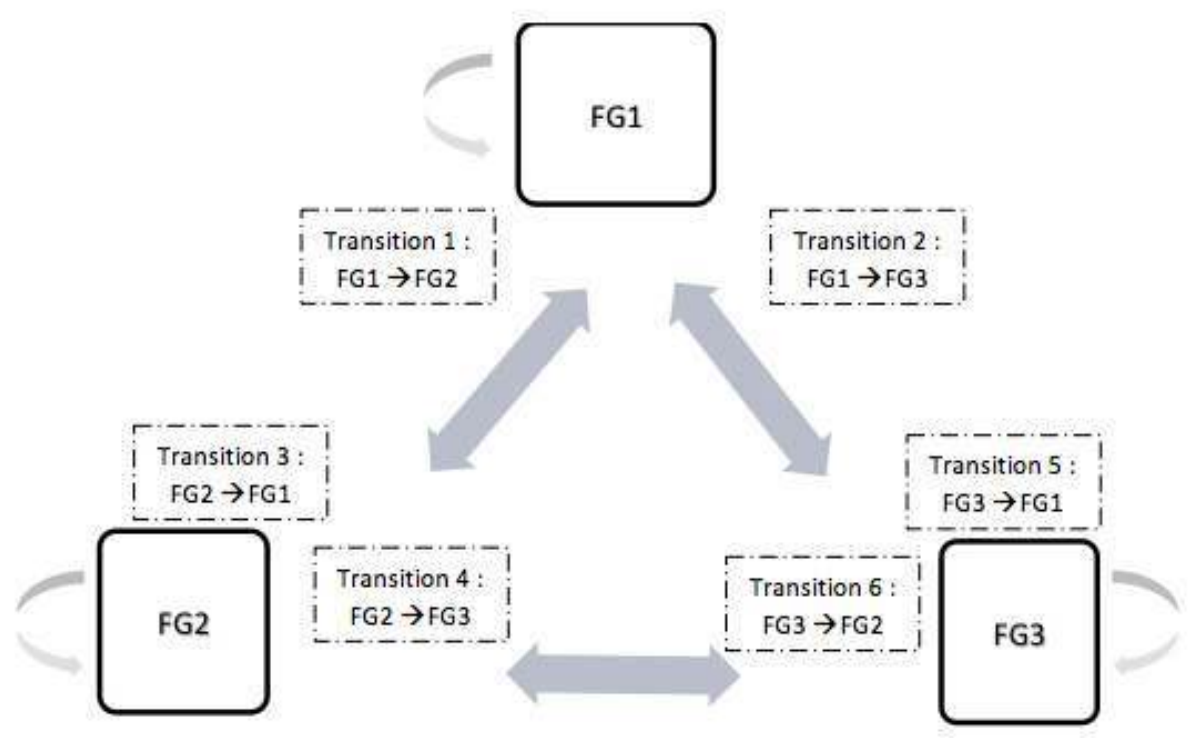

\section{2. Échantillon et sources des données}

Nous avons testé les hypothèses proposées dans cette recherche sur un échantillon constitué de $118^{12}$ les plus grandes entreprises françaises en termes de capitalisation boursière et de volumes d'échanges sur le marché Euronext Paris, appartenant ou ayant appartenu à l'indice SBF 120 sur la période 2000-2014. Les données sont issues de la base de données IODS et ont été vérifiées ou complétées par une recherche manuelle dans les documents de référence AMF des entreprises quand cela été nécessaire.

\subsection{Opérationnalisation des variables}

\subsubsection{Variable dépendante}

Le phénomène que nous cherchons à expliquer est la durée des différents épisodes ainsi que l'intensité et la nature des transitions d'une forme de gouvernance à une autre. La variable expliquée est donc la durée des formes de gouvernance des entreprises françaises cotées. Le tableau 1 ci-dessous décrit la représentativité des transitions observées et renseignées dans la base de données. On comptabilise une transition $\mathrm{i} \rightarrow \mathrm{j}$ quand on observe une entreprise dans l'état $i$ une année donnée et qu'elle se trouve dans l'état $\mathrm{j}$ l'année suivante $(i, j=1, \ldots, 3)$. Dans de nombreux cas, on n'observe pas de changement d'état d'une année à l'autre (transition i $\rightarrow$ i) : parmi les 118 entreprises étudiées, $29 \%$ connaissent uniquement la forme de gouvernance FG1, $3 \%$ que la FG2 et $16 \%$ que la FG3. On dénombre un plus grand nombre de passages de FG1 $\rightarrow \mathrm{FG} 2(32 \%)$ et $\mathrm{FG} 2 \rightarrow \mathrm{FG} 1(16 \%)$ que de $\mathrm{FG} 1 \rightarrow \mathrm{FG} 3(8 \%)$ et $\mathrm{FG} 3 \rightarrow \mathrm{FG} 2(6 \%)$. 
Tableau 2. Dynamique des changements de la forme de gouvernance

\begin{tabular}{|c|c|c|c|c|c|c|c|c|}
\hline $\begin{array}{l}\text { Type de } \\
\text { transition }\end{array}$ & & $\begin{array}{l}\text { Effectif } \\
\text { avec } \\
\text { transition } \\
\text { potentielle }\end{array}$ & $\begin{array}{l}\text { Effectif } \\
\text { avec } \\
\text { transition } \\
\text { avérée* }\end{array}$ & $\begin{array}{l}\text { Pourcentage } \\
\text { avec } \\
\text { transition } \\
\text { avérée }\end{array}$ & $\begin{array}{l}\text { durée } \\
\text { moyenne }\end{array}$ & $\begin{array}{l}\text { durée } \\
\text { minimum }\end{array}$ & $\begin{array}{l}\text { durée } \\
\text { médiane }\end{array}$ & $\begin{array}{l}\text { durée } \\
\text { maximum }\end{array}$ \\
\hline $\mathrm{FG} 1>\mathrm{FG} 2$ & $\begin{array}{l}\text { Transition } \\
1\end{array}$ & 91 & 38 & $33 \%$ & 9,4 & 1 & 9 & 14 \\
\hline FG1->FG3 & $\begin{array}{l}\text { Transition } \\
2\end{array}$ & 91 & 9 & $8 \%$ & 9,4 & 1 & 9 & 14 \\
\hline $\mathrm{FG} 2->\mathrm{FG} 1$ & $\begin{array}{l}\text { Transition } \\
3\end{array}$ & 42 & 21 & $18 \%$ & 6,1 & 1 & 6 & 12 \\
\hline FG2->FG3 & $\begin{array}{l}\text { Transition } \\
4\end{array}$ & 42 & 0 & $0 \%$ & 0 & 0 & 0 & 0 \\
\hline FG3->FG1 & $\begin{array}{l}\text { Transition } \\
5\end{array}$ & 45 & 16 & $14 \%$ & 10,8 & 1 & 13 & 14 \\
\hline FG3 $>>$ FG2 & $\begin{array}{l}\text { Transition } \\
6\end{array}$ & 45 & 7 & $6 \%$ & 10,8 & 1 & 13 & 14 \\
\hline FG1-FG1 & $\begin{array}{l}\text { Pas de } \\
\text { transition }\end{array}$ & & 34 & $30 \%$ & 9,4 & 1 & 9 & 14 \\
\hline FG2-FG2 & $\begin{array}{l}\text { Pas de } \\
\text { transition }\end{array}$ & & 3 & $3 \%$ & 6,1 & 1 & 6 & 12 \\
\hline FG3-FG3 & $\begin{array}{l}\text { Pas de } \\
\text { transition }\end{array}$ & & 19 & $17 \%$ & 10,8 & 1 & 13 & 14 \\
\hline
\end{tabular}

Note : FG1 - CA avec PDG ; FG2 - CA avec séparation des fonctions ; FG3 - Directoire et Conseil de surveillance.

*Certaines entreprises ont connu plusieurs types de transition, donc le nombre total de transitions est supérieur au nombre d'entreprises dans l'échantillon des 118 entreprises retenues.

\subsubsection{Variables indépendantes}

19 Nos hypothèses suggèrent que deux séries de facteurs essentiels sont susceptibles d'influer sur la durée et la forme de gouvernance. La première série de facteurs est relative à la gouvernance de l'entreprise (dirigeant, conseil, actionnariat) et la seconde série renvoie à des facteurs « de contingence » (performance de l'entreprise et secteur d'activité). Les variables décrites ci-dessous sont retardées d'une année afin de limiter pour partie les problèmes d'endogénéité potentielle. En ce qui concerne le dirigeant ${ }^{13}$, nous avons considéré que l'âge du dirigeant - exprimé en nombre d'années pour l'année considérée (Belot et al. 2014) - était un bon proxy qui permet en grande partie de capter la stratégie d'enracinement de ce dernier (Hollandts et al. 2015; Belot et al. 2014 ; Raheja 2005). En ce qui concerne l'actionnariat, nous avons inclus une série de variables 
permettant de mesurer la concentration (pourcentage de capital détenu par le premier actionnaire) et l'identité des principaux actionnaires. Par ailleurs, nous avons également inclus une série de variables binaires permettant d'identifier le type de premier actionnaire (État, famille, autre entreprise, institution financière-banque, assurances, fonds d'investissement). Enfin, nous avons inclus le pourcentage de capital détenu par les salariés, car la littérature souligne que les salariés sont en mesure de passer des contrats implicites et avec leurs dirigeants notamment (Faleye, Merhotra et Morck 2006), leur permettant de résister aux tentatives de changement de structure. Nos modèles incluent également la proportion de femmes au sein du conseil (ratio rapportant le nombre d'administratrices sur la taille du conseil - Belot et al. 2014). Une seconde série de facteurs dits « de contingence » sont relatifs à l'environnement et à la performance de l'entreprise. La performance de l'entreprise est mesurée par le rendement des capitaux propres calculé comme le résultat net sur les capitaux propres (Linck et al. 2008). Nous avons également inclus une mesure indirecte de la performance de l'entreprise avec le ratio (chiffres d'affaires/nombre de salariés) qui est un proxy généralement utilisé pour mesurer la productivité des salariés. Enfin, nous avons inclus une mesure de l'endettement de l'entreprise.

\subsubsection{Variables de contrôle}

20 Nos modèles statistiques incorporent une série de variables de contrôle qui peuvent influencer la décision de changement de structure de gouvernance. Plus précisément, nous avons inclus des variables contrôlant les effets du secteur d'activité, de la taille de l'entreprise et l'année 2001 (loi NRE). L'environnement concurrentiel est susceptible d'avoir une influence sur le choix de la structure de gouvernance (Fama et Jensen 1983). Nous avons utilisé sept variables binaires représentatives du code sectoriel principal de chacune des entreprises. Par ailleurs, la taille de l'entreprise (logarithme du chiffre d'affaires) est souvent corrélée au champ et à la complexité de l'environnement (Linck et al. 2008 ; Boone et al. 2007). Enfin, nous avons inclus une variable muette pour l'année 2001 afin de prendre en considération les effets de la loi NRE sur les transitions vers d'autres formes de gouvernance. Les statistiques descriptives et les corrélations entre les variables sont présentées en annexe 1.

\section{Résultats}

Les résultats des différentes estimations correspondantes aux différentes lois de distribution combinées aux différents types de transition sont présentés ci-après dans le tableau 3. Le tableau ci-après présente en tout 6 modèles. Le premier porte sur l'échantillon total (et donc l'ensemble des transitions potentielles) tandis que les 5 modèles suivants s'attachent à observer l'effet différencié des variables suivant le type de transition observée. 
Tableau 3. Résultats des régressions des modèles multi-états testant les effets des variables de gouvernance et de performance sur la durée et le type de transition observée

\begin{tabular}{|c|c|c|c|c|c|c|}
\hline Modèle & $\begin{array}{l}1 \\
\text { (Ech. total) }\end{array}$ & $\begin{array}{l}2 \\
\text { (Sous-éch.) }\end{array}$ & $\begin{array}{l}3 \\
\text { (Sous-éch.) }\end{array}$ & $\begin{array}{l}4 \\
\text { (Sous- } \\
\text { éch.) }\end{array}$ & $\begin{array}{l}5 \\
\text { (Sous-éch.) }\end{array}$ & $\begin{array}{l}6 \\
\text { (Sous-éch.) }\end{array}$ \\
\hline & & Transition1 & Transition2 & $\begin{array}{l}\text { Transition } \\
3\end{array}$ & Transition5 & Transition6 \\
\hline & & FG1 à FG2 & FG1 à FG3 & FG2 à FG1 & FG3 à FG1 & FG3 à FG2 \\
\hline $\begin{array}{ll}\text { Type de } \\
\text { distribution }\end{array}$ & $\begin{array}{l}\text { Distrib. } \\
\text { Exp. Avec } \\
\text { frailty }\end{array}$ & $\begin{array}{l}\text { Distrib. } \\
\text { Exp. }\end{array}$ & $\begin{array}{l}\text { Distrib. } \\
\text { Gompertz }\end{array}$ & $\begin{array}{l}\text { Distrib. } \\
\text { Exp. }\end{array}$ & $\begin{array}{l}\text { Distrib. } \\
\text { Weibull }\end{array}$ & $\begin{array}{l}\text { Distrib. } \\
\text { Gompertz }\end{array}$ \\
\hline & $\begin{array}{l}\text { Likelihood } \\
\text { ratio } \\
\text { (Proba) }\end{array}$ & $\begin{array}{l}\text { Likelihood } \\
\text { ratio } \\
\text { (Proba) }\end{array}$ & $\begin{array}{l}\text { Likelihood } \\
\text { ratio } \\
\text { (Proba) }\end{array}$ & $\begin{array}{l}\text { Likelihood } \\
\text { ratio } \\
\text { (Proba) }\end{array}$ & $\begin{array}{l}\text { Likelihood } \\
\text { ratio } \\
\text { (Proba) }\end{array}$ & $\begin{array}{l}\text { Likelihood } \\
\text { ratio } \\
\text { (Proba) }\end{array}$ \\
\hline boucle & $\begin{array}{l}9,21^{* * *} \\
(0,00)\end{array}$ & $\begin{array}{l}5,43^{* * *} \\
(0,00)\end{array}$ & $\begin{array}{l}2,91 \\
(0,34)\end{array}$ & & & \\
\hline trans_1 & $\begin{array}{l}2,23 \\
(0,12)\end{array}$ & & & & & \\
\hline trans_2 & $\begin{array}{l}0,45 \\
(0,21)\end{array}$ & & & & & \\
\hline trans_3 & $\begin{array}{l}12,59^{* * *} \\
(0,00)\end{array}$ & & & & & \\
\hline trans_5 & $\begin{array}{l}4,49^{* * *} \\
(0,01)\end{array}$ & & & & & \\
\hline Année 2001 & $\begin{array}{l}1,17 \\
(0,79)\end{array}$ & $\begin{array}{l}2,54^{*} \\
(0,07)\end{array}$ & $\begin{array}{l}1.51 \\
(0.66)\end{array}$ & $\begin{array}{l}0.00^{* * *} \\
(0.00)\end{array}$ & $\begin{array}{l}0.00^{* * *} \\
(0.00)\end{array}$ & $\begin{array}{l}0.00^{* * *} \\
(0.00)\end{array}$ \\
\hline Performance & $\begin{array}{l}1.02^{*} \\
(0.09)\end{array}$ & $\begin{array}{l}1.03^{*} \\
(0.05)\end{array}$ & $\begin{array}{l}1.05 \\
(0.46)\end{array}$ & $\begin{array}{l}1.02 \\
(0,43)\end{array}$ & $\begin{array}{l}1,01 \\
(0,85)\end{array}$ & $\begin{array}{l}1,04 \\
(0,66)\end{array}$ \\
\hline CA/actions & $\begin{array}{l}1,00 \\
(0,17)\end{array}$ & $\begin{array}{l}0,99 * \\
(0,06)\end{array}$ & $\begin{array}{l}0,96 \\
(0,26)\end{array}$ & $\begin{array}{l}1,01 \\
(0,46)\end{array}$ & $\begin{array}{l}1,02 \\
(0,19)\end{array}$ & $\begin{array}{l}1,03 \\
(0,18)\end{array}$ \\
\hline $\begin{array}{l}\text { Concentration } \\
\text { actionnariat }\end{array}$ & $\begin{array}{l}1,01 \\
(0,43)\end{array}$ & $\begin{array}{l}1,00 \\
(0,70)\end{array}$ & $\begin{array}{l}0,98 \\
(0,40)\end{array}$ & $\begin{array}{l}0,98^{*} \\
(0,07)\end{array}$ & $\begin{array}{l}1,00 \\
(0,97)\end{array}$ & $\begin{array}{l}1,06^{*} \\
(0,07)\end{array}$ \\
\hline
\end{tabular}




\begin{tabular}{|c|c|c|c|c|c|c|}
\hline $\begin{array}{l}\text { Actionnariat } \\
\text { salarié }\end{array}$ & $\begin{array}{l}0,97 \\
(0,37)\end{array}$ & $\begin{array}{l}0,95^{*} \\
(0,09)\end{array}$ & $\begin{array}{l}0,78 \\
(0,33)\end{array}$ & $\begin{array}{l}1,06 \\
(0,63)\end{array}$ & $\begin{array}{l}1,18^{* * *} \\
(0,01)\end{array}$ & $\begin{array}{l}1,01 \\
(0,90)\end{array}$ \\
\hline État & $\begin{array}{l}0,89 \\
(0,79)\end{array}$ & $\begin{array}{l}0,77 \\
(0,72)\end{array}$ & $\begin{array}{l}1,44 \\
(0,77)\end{array}$ & $\begin{array}{l}0,31 \\
(0,16)\end{array}$ & $\begin{array}{l}0,23 * \\
(0,10)\end{array}$ & $\begin{array}{l}81,48^{* * *} \\
(0,00)\end{array}$ \\
\hline Entreprise & $\begin{array}{l}0,56 \\
(0,35)\end{array}$ & $\begin{array}{l}0,50 \\
(0,24)\end{array}$ & $\begin{array}{l}0,41 \\
(0,61)\end{array}$ & $\begin{array}{l}1,22 \\
(0,83)\end{array}$ & $\begin{array}{l}1,22 \\
(0,85)\end{array}$ & $\begin{array}{l}1,86 \\
(0,61)\end{array}$ \\
\hline Famille & $\begin{array}{l}0,39 * * \\
(0,05)\end{array}$ & $\begin{array}{l}0,32^{*} \\
(0,06)\end{array}$ & $\begin{array}{l}0,36 \\
(0,32)\end{array}$ & $\begin{array}{l}0,19^{* * *} \\
(0,00)\end{array}$ & $\begin{array}{l}0,38 \\
(0,19)\end{array}$ & $\begin{array}{l}0,68 \\
(0,73)\end{array}$ \\
\hline $\begin{array}{l}\% \text { de femmes } \\
\text { au conseil }\end{array}$ & $\begin{array}{l}0,98^{*} \\
(0,07)\end{array}$ & $\begin{array}{l}0,99 \\
(0,44)\end{array}$ & $\begin{array}{l}1,01 \\
(0,66)\end{array}$ & $\begin{array}{l}1,01 \\
(0,70)\end{array}$ & $\begin{array}{l}0,99 \\
(0,83)\end{array}$ & $\begin{array}{l}0,94 \\
(0,28)\end{array}$ \\
\hline Âge dirigeant & $\begin{array}{l}1,09^{* * *} \\
(0,00)\end{array}$ & $\begin{array}{l}1,19^{* * *} \\
(0,00)\end{array}$ & $\begin{array}{l}1,19^{* *} \\
(0,02)\end{array}$ & $\begin{array}{l}0,94^{* *} \\
(0,05)\end{array}$ & $\begin{array}{l}1,03 \\
(0,45)\end{array}$ & $\begin{array}{l}1,00 \\
(0,96)\end{array}$ \\
\hline $\begin{array}{l}\text { Actifs } \\
\text { intangibles }\end{array}$ & $\begin{array}{l}1,00 \\
(0,14)\end{array}$ & $\begin{array}{l}1,00 \\
(0,30)\end{array}$ & $\begin{array}{l}1,00^{* * *} \\
(0,00)\end{array}$ & $\begin{array}{l}1,00^{* *} \\
(0,04)\end{array}$ & $\begin{array}{l}1,00^{* * *} \\
(0,01)\end{array}$ & $\begin{array}{l}1,00 \\
(0,81)\end{array}$ \\
\hline Endettement & $\begin{array}{l}1,00^{*} \\
(0,09)\end{array}$ & $\begin{array}{l}1,00 \\
(0,45)\end{array}$ & $\begin{array}{l}1,00 \\
(0,96)\end{array}$ & $\begin{array}{l}0,99 \\
(0,63)\end{array}$ & $\begin{array}{l}1,00 \\
(0,65)\end{array}$ & $\begin{array}{l}1,00 \\
(0,13)\end{array}$ \\
\hline $\begin{array}{l}\text { Chiffre } \\
\text { d'affaires }\end{array}$ & $\begin{array}{l}1,00 \\
(0,19)\end{array}$ & $\begin{array}{l}1,00 \\
(0,25)\end{array}$ & $\begin{array}{l}1,00^{* *} \\
(0,01)\end{array}$ & $\begin{array}{l}1,00 \\
(0,88)\end{array}$ & $\begin{array}{l}1,00^{*} \\
(0,05)\end{array}$ & $\begin{array}{l}1,00^{* * *} \\
(0,00)\end{array}$ \\
\hline Productivité & $\begin{array}{l}1,00 \\
(0,22)\end{array}$ & $\begin{array}{l}1,00 \\
(0,25)\end{array}$ & $\begin{array}{l}1,00^{* *} \\
(0,04)\end{array}$ & $\begin{array}{l}1,00 \\
(0,74)\end{array}$ & $\begin{array}{l}1,00 \\
(0,14)\end{array}$ & $\begin{array}{l}1,00^{* * *} \\
(0,00)\end{array}$ \\
\hline Constant & $\begin{array}{l}0,00^{* * *} \\
(0,00)\end{array}$ & $\begin{array}{l}0,00^{* * *} \\
(0,00)\end{array}$ & $\begin{array}{l}1,5 \mathrm{e}+169^{* * *} \\
(0,00)\end{array}$ & $\begin{array}{l}1,75 \\
(0,77)\end{array}$ & $\begin{array}{l}0,00 \\
(0,15)\end{array}$ & $\begin{array}{l}0,00 \\
(0,55)\end{array}$ \\
\hline $\begin{array}{l}\text { Secteurs (SIC } \\
\text { code) }\end{array}$ & Oui & Oui & Oui & Oui & Oui & Oui \\
\hline Observations & 2858 & 818 & 818 & 232 & 379 & 379 \\
\hline $\begin{array}{l}\mathrm{Nb} \\
\text { d'entreprises }\end{array}$ & 118 & 91 & 91 & 42 & 45 & 45 \\
\hline chi2 & $124,84^{* * *}$ & $169,09^{* * *}$ & $2078,61^{* * *}$ & $1013,37^{* * *}$ & $4211,67^{* * *}$ & $6640,04^{* * *}$ \\
\hline aic & $-304,63$ & $-135,56$ & 10,34 & $-102,60$ & $-27,67$ & 3,02 \\
\hline bic & $-161,56$ & $-45,95$ & 104,67 & $-41,27$ & 47,45 & 78,13 \\
\hline
\end{tabular}


Note : La transition 4 de FG2 vers FG3 n'a pas d'occurrence dans notre échantillon (cf. Tableau 1).

\section{Interprétation des résultats}

Les estimations présentées dans le tableau 3 portent à la fois sur l'échantillon total et également sur les différents types de transitions observées (modèles 2 à 6). Le tableau 3 peut être lu et interprété de deux façons. D'une part, colonne par colonne en comparant l'effet d'une même variable suivant les différents types de transition. D'autre part, ligne par ligne en examinant l'effet d'une même variable suivant les types de transitions. Dans ce cas, on peut isoler l'effet de chaque variable suivant le type de transition observée alors que l'effet global de cette variable n'est pas nécessairement perceptible sur l'échantillon total. L'interprétation des facteurs est particulière dans le sens où l'on doit interpréter la déviation par rapport à 1 . Deux exemples concrets permettent de saisir comment interpréter les résultats du tableau 3.

$1.09^{* * *}$ : si la variable $\mathrm{X}$ augmente, alors la durée dans cette forme de gouvernance augmente = la transition de $\mathrm{FG}_{\mathrm{i}}$ vers $\mathrm{FG}_{\mathrm{j}}$ est retardée lorsque $\mathrm{X}$ augmente. En d'autres termes, un signe supérieur à 1 a une influence positive sur la durée et le maintien dans la forme de gouvernance observée.

$0.98^{*}$ : si la variable $\mathrm{X}$ augmente, alors la durée dans cette forme de gouvernance diminue (inférieur à 1 ) = la transition de $F_{i}$ vers $F_{j}$ est accélérée lorsque $X$ augmente. En d'autres termes, un signe inférieur à 1 a une influence négative sur la durée et le maintien dans la forme de gouvernance observée.

\section{Les résultats par estimations et type de transition}

Pour plus de simplicité, les résultats sont interprétés modèle par modèle. Dans le modèle 1 (échantillon total), on interprète l'influence des variables indépendantes sur la durée dans une forme de gouvernance, quel que soit son type. Les résultats de ce modèle sont " globaux » et sont relativement peu intéressants, car ils masquent ce qui se passe selon le type de transition. Les variables trans nous renseignent sur la durée des états selon les types de transitions. Ainsi, les transitions 3 et 5 (passage d'une structure dissociée ou duale à la forme PDG) sont significatives. Cela nous indique que ce type de transitions se produit au bout d'une durée significativement plus importante que pour l'ensemble des transitions. Parmi les variables de ce modèle, on peut relever l'influence significative de la performance, qui, ceteris paribus, tend à augmenter la durée d'une forme de gouvernance donnée. Ce résultat est attendu dans le sens où une bonne performance de l'entreprise tend à maintenir, toutes choses égales par ailleurs, la gouvernance en place. Deuxième variable significative, la présence d'un actionnariat familial significatif a une influence sur la durée de la gouvernance. Ce résultat peut s'expliquer par la spécificité des entreprises familiales qui tendent à faire évoluer leur forme de gouvernance afin de faciliter les processus de transition en leur sein (Charlier et Lambert 2013). Autre variable significative, plus l'âge du dirigeant augmente et plus la durée de la forme de gouvernance tend à s'accroître. Il faut sans doute y voir l'influence du départ du dirigeant en raison de la proximité de la retraite (Marcel et al. 2017). Enfin, le pourcentage de femmes au conseil a également une influence significative ce qui dénote des changements liés sans doute à la féminisation du conseil suite à la loi dite Copé/Zimmerman (2011), les 
conseils ayant dû faire évoluer leurs structures de gouvernance en se mettant en conformité au regard de cette nouvelle législation (Allemand et Brullebaut 2014 ; Gregoir et al. 2013).

Le reste des modèles testés nous permet d'avoir une approche différenciée et « comparative " selon le type de transition, qui nous semble a priori plus intéressante à détailler. Comme dans le modèle général (échantillon total), les estimations permettent à la fois d'analyser la durée et le type de transition ultérieure. En ce qui concerne les transitions pour les entreprises ayant la forme "CA avec PDG » on constate qu'elles peuvent aller vers la forme CA avec P-DG ou la structure duale. Comme dans le modèle global, la performance a un effet significatif et tend à favoriser le maintien de la forme " CA avec PDG ». L'âge du dirigeant a également un impact positif et significatif sur le maintien de cette forme.

Les modèles $2 \& 3$ permettent de décomposer les deux types de transition en partant de la forme " CA avec PDG». La performance tend à augmenter le maintien de la forme " CA avec PDG» seulement dans les transitions potentielles vers la seconde forme (séparation des fonctions de P-DG). Par ailleurs, on constate un effet significatif et négatif lorsque le premier actionnaire est familial. Dans ce type de transition (du « CA avec PDG » au « CA avec P-DG»), le fait d'avoir une famille comme premier actionnaire favorise une transition vers la forme « CA avec P-DG». Ceci peut s'expliquer par les cas de transitions au sein des entreprises familiales qui se concrétisent par la nomination potentielle de l'ancien DG exécutif au poste du président du conseil tandis que son successeur prend le poste de DG (Charlier et Lambert 2013).

Pour le second type de transition en partant de la forme « CA avec PDG » vers la structure duale (modèle 3), on peut noter l'effet significatif de certaines variables. La transition potentielle vers une structure duale est négativement liée à la taille de l'entreprise. Dit autrement, ce type de transition est moins fréquent dans les très grandes entreprises. Dans les modèles 2 et 3, l'âge du dirigeant a un effet négatif, tendant à maintenir ces entreprises dans la forme " CA avec PDG ». Plus le dirigeant est âgé et plus la probabilité que l'entreprise connaisse une transition est faible. D'un point de vue technique, le paramètre gamma (non reporté dans le tableau 3 ), est significatif et positif $\left(0,82^{* * *}\right)$, ce qui implique que plus une entreprise a passé de temps dans la forme « $C A$ avec PDG » et plus elle a de chance de passer à une structure duale, toutes choses égales par ailleurs ${ }^{14}$.

Le modèle 4 explique la durée passée dans la forme " CA avec P-DG ». Ce sous-échantillon correspond aux entreprises qui sont observées dans cette forme et qui opèrent une transition (un retour?) vers la forme "CA avec PDG». Cette estimation comporte plusieurs effets qui se distinguent des autres estimations réalisées. La transition voire le retour vers la forme « CA avec PDG » est favorisée par la concentration de l'actionnariat et la présence d'un actionnariat familial significatif. On peut y voir l'effet des stratégies de transitions notamment au sein d'entreprises familiales, qui consistent à revenir à une forme moniste unifiée une fois la transition achevée (Charlier et Lambert 2013). Sans doute, l'organisation de la succession à la tête des entreprises nécessite un aménagement de la forme de gouvernance afin de revenir vers la forme moniste habituelle. Une autre explication pourrait être liée à la volonté de renforcer l'attractivité de la gouvernance en renforçant les pouvoirs du PDG, notamment dans la perspective de recrutements extérieurs. 
, les modèles 5 et 6 examinent les effets des transitions potentielles en partant de la structure duale. L'estimation du paramètre gamma d'influence du temps passé dans une forme duale n'est pas significativement différente de zéro (1,11 non reporté dans le tableau 3). Le temps n'exerce pas d'influence indépendante sur la probabilité de transition vers une autre forme, à la différence de la forme "CA avec PDG ». Ces résultats peuvent être expliqués par le fait que lorsque l'on décompose les estimations en transitions plus fines, deux lois différentes s'appliquent pour le passage de FG3 à FG1 et de FG3 à FG2. Le modèle 5 montre que la présence de salariés au capital a une influence positive sur la transition vers une forme moniste. Deux explications peuvent être avancées : soit les actionnaires salariés ont une préférence pour ce type de gouvernance unifiée, soit ils sont " instrumentalisés " par les dirigeants en place, qui ont en général intérêt à évoluer au sein d'une structure moniste et si possible avec la fonction unifiée de PDG (Krause 2017 ; Faleye et al. 2006). En ce qui concerne le modèle 6, le passage d'une structure duale vers la forme " $\mathrm{CA}$ avec P-DG» est retardé par la concentration de l'actionnariat, la présence significative de l'État au capital et la taille de l'entreprise. En revanche, on ne relève pas d'influence de l'actionnariat salarié en ce qui concerne ce type de transition.

\section{L'influence des variables dans les différentes estimations}

Une interprétation « ligne par ligne » nous offre une lecture complémentaire de l'effet de certaines variables. L'effet significatif de la performance est uniquement observable dans les transitions concernant les entreprises partant d'une forme " CA avec PDG». Cette forme dominante parmi les entreprises cotées (environ 2/3 des entreprises en moyenne selon les années) tend à rester stable dans le temps lorsque la performance est bonne. En revanche, on ne constate pas d'effet significatif sur les autres formes de transition (modèles 3 à 6).

Du point de vue de la gouvernance, la variable ayant une influence quasi systématique est l'âge du dirigeant qui tend à stabiliser la forme " CA avec PDG », mais tend à favoriser les transitions quand l'entreprise part de la forme " CA avec P-DG». La combinaison de la proximité de la retraite des dirigeants et de la forme de la gouvernance semble avoir un effet significatif sur la probabilité de transition vers d'autres formes de gouvernance. Autre effet détecté par nos estimations, la proportion des femmes au conseil est significative dans le modèle global (modèle 1), mais s'avère non significative quand on décompose par type de transition. Cet effet global est sans doute lié à des raisons d'ajustement et de conformité à la loi Copé/Zimmerman (Allemand et Brullebaut 2014). Du point de vue des variables de contrôle, on peut noter que plus la taille de l'entreprise est importante et plus la probabilité de transition diminue. Les très grandes entreprises ont donc une gouvernance plus stable dans le temps que les autres entreprises. Pris ensemble, ces différents résultats mettent en exergue des facteurs essentiels de la stabilisation de la gouvernance des sociétés cotées. Ainsi, les plus grandes entreprises et les plus performances ont (logiquement?) moins de chances de changer de formes de gouvernance (Boyd et al. 2011 ; Bebchuck et Weisbach 2010). La combinaison de la taille et de la performance plaiderait pour un maintien de la coalition en place (dirigeants, administrateurs, actionnaires) (Aguilera et al. 2008; Belot et al. 2014 ; Krause 2017). Cet effet majeur peut être soutenu par d'autres facteurs de stabilisation comme la concentration de l'actionnariat (Mard et al. 2014). Par contraste, on peut suggérer que les sociétés d'une taille plus modeste, qui ne disposent pas d'un capital concentré et affichent

Finance Contrôle Stratégie, 21-3 | 2018 
des performances plus faibles ont plus de chances de voir leur structure de gouvernance évoluer. On peut également noter que l'identité des actionnaires joue un rôle important en favorisant (famille, salariés) ou en retardant (Etat) les transitions vers d'autres formes de gouvernance.

\section{Discussion}

31 L'objectif de cette recherche était d'examiner les facteurs ayant un impact sur la durée et la stabilité des structures de gouvernance des sociétés cotées en France. Examiner l'influence de certains facteurs sur le choix de la dernière structure de gouvernance n'est pas nouveau dans le contexte français (Belot et al. 2014). En revanche, notre article entend compléter la littérature existante en examinant de façon détaillée la dynamique de changement de la gouvernance des sociétés. À notre connaissance, la littérature s'est très peu intéressée à la question de la stabilité dynamique de la gouvernance des entreprises à l'exception de Kole et Lehn (1999 \& 1997). Dans cet article, nous ne considérons pas seulement l'influence ponctuelle de certaines variables sur un choix précis, à un instant donné, mais envisageons les changements de structure de gouvernance comme un processus dynamique et de long terme (Marcel et al. 2017 ; Joseph et al. 2014 ; Boone et al. 2007), répondant en cela à un appel de la communauté académique (Boivie et al. 2016 ; Wintoki et al. 2012). En général, la littérature académique se concentre seulement sur la dernière forme connue sans tenir compte de " l'historique » de l'entreprise en la matière (Boyd et al. 2017; Krause 2017), ce qui n'offre qu'un aperçu partiel des processus et de l'efficacité de la gouvernance de l'entreprise. Or, les changements observables de structures de gouvernance sont très souvent la résultante de processus de long terme qui nécessitent dès lors un appareillage théorique et méthodologique qui permet de prendre en compte la spécificité de ces données (Marcel et al. 2017) et c'est que nous sommes efforcés de réaliser dans le cadre de cet article. Notre étude porte sur un échantillon (SBF 120) communément mobilisé pour analyser la gouvernance des sociétés cotées françaises et sur une période temporelle importante (2000-2014), qui englobe notamment la période de " diffusion" des principaux textes législatifs (loi NRE) et codes de gouvernance français (code Afep-Medef essentiellement) voire étrangers (recommandations de la Commission Européenne ou les Principes de l'OCDE par exemple) ${ }^{15}$. Les données que nous avons utilisées sont plus récentes que celles mobilisées par Belot et al. (2014) ou Hollandts et al. (2015) qui s'achevaient en 2008. De notre côté, nous avons pu travailler sur une période relativement longue (15 années) et sur des données plus récentes (2000/2014).

Nos contributions peuvent globalement être situées à deux niveaux. D'une part, nous avons pu étudier la dynamique du changement de structure et pas seulement l'impact « ponctuel » de certaines variables sur le choix de la structure de gouvernance. En cela, nous intégrons à la différence de ces deux articles, l'influence du temps passé et du type de structure de gouvernance qui constituent deux éléments déterminants dans la stabilité et le maintien d'une forme de gouvernance donnée (au détriment des deux autres formes donc). Belot et al. (2014) comme Hollandts et al. (2015) ne considéraient quant à eux que l'effet des variables sur le choix de la dernière structure connue. D'autre part, notre article se focalise également sur le type de transition et examine ainsi au travers de souséchantillons, l'effet différencié de nos variables indépendantes selon le type de transition observée. On peut analyser cela comme un second aspect de la dimension dynamique. En effet, les chemins transitionnels ne sont pas équivalents. Passer d'une forme « $\mathrm{CA}$ avec 
PDG » à une structure duale est une transition moins évidente que de simplement séparer les fonctions de PDG, tout en conservant la forme CA. Les différents résultats montrent à la fois que le temps passé dans une forme a une influence sur la propension à changer ou non (variables trans) et l'analyse « ligne par ligne » nous indique que certaines variables ont un effet différencié selon le type de transition observée. D'un point de vue économétrique, notre approche, qui mobilise des modèles multi-états en panel de type semi-markovien est donc différente de celles qui sont utilisées par Belot et al. (modèle logistique binomial) ou par Hollandts et al. (stereotype logit) qui ne considèrent en somme que l'influence des variables sur un choix binaire (CA ou une structure duale) ou un choix binomial (deux modalités de CA et la structure duale), et seulement sur la dernière forme de gouvernance observée.

Nos résultats, analysés de façon globale, peuvent être résumés autour de trois éléments principaux.

Premièrement, on constate l'existence d'une prime à la stabilité. Cela est notamment vrai pour la forme CA, qui est majoritaire parmi les sociétés de notre échantillon et qui a tendance à connaître moins de transitions que les autres formes. Ainsi, il existe un différentiel de 6 points entre les transitions de la forme CA vers la structure duale et l'inverse ( $8 \%$ contre $14 \%)$. Par ailleurs, nos résultats montrent que la performance contribue à maintenir cette forme de gouvernance, alors que l'effet n'est pas perceptible pour les autres formes. Ainsi les facteurs de contingence (Aguilera et al. 2008) sont susceptibles de jouer un rôle important. L'examen des variables de contrôle tendent à montrer les plus grandes entreprises affichent une gouvernance plus stable que les autres entreprises de moindre taille composant l'échantillon (impact significatif dans les modèles 3, 5 et 6). Au final, un portrait-robot de la gouvernance stable se dessine : une grande entreprise cotée, affichant de bonnes performances et ayant adopté en général la forme du conseil d'administration.

Nos résultats mettent par ailleurs en exergue des facteurs ayant une influence sur les transitions entre les différentes formes de gouvernance. On peut notamment noter l'influence déterminante de l'âge du dirigeant. Une grande partie des stratégies d'enracinement des dirigeants sont liées à leur perspective d'enracinement lié à leur volonté de s'assurer de leur maintien en poste jusqu'à la retraite (Krause 2017). Ce résultat complète ceux obtenus par Hollandts et al. (2015) qui avaient montré que la durée en poste du dirigeant avait un impact négatif sur la probabilité de changement de structure. La présence de cette variable dans nos estimations nous permet de mettre en exergue des effets très significatifs dans la majorité des modèles. L'âge du dirigeant est positivement lié à la stabilité de la structure de gouvernance dans nos quatre premières estimations, qui concernent à la fois l'échantillon total (toutes transitions confondues) et les sous-échantillons relatifs à la forme "CA avec PDG». Élément intéressant, cette variable est également significative, mais négative dans notre modèle 4 qui se focalise sur les transitions des entreprises partant de la forme " CA avec P-DG ». Sans doute faut-il y voir l'effet contrasté de la proximité de l'âge de la retraite. Dans le premier cas, le PDG, sans doute en mesure de dominer son conseil (Krause 2017 ; Joseph et al. 2014) s'assure de rester en poste et la relation positive observée témoigne de la stabilité de la structure, mais également de son maintien en poste. Dans le cas du modèle 4 (entreprise partant de la forme " CA avec P-DG»), les successions potentielles (plus aisées avec la forme "CA avec P-DG») peuvent expliquer la relation négative constatée. Dans ce cas, l'âge du dirigeant est négativement lié à la durée de la structure et l'effet observé peut témoigner 
de successions potentielles. Une fois le successeur nommé, les entreprises partant de cette forme peuvent adopter une structure duale ou bien revenir à la forme moniste pure (CA avec PDG). En ce qui concerne la structure actionnariale, on peut notamment relever l'influence contrastée de l'actionnariat salarié qui tend à réduire la stabilité des entreprises ayant une forme moniste, mais qui stabilise les entreprises partant d'une structure duale. Un examen des autres types d'actionnaires montre que la présence d'un actionnariat familial tend à faire évoluer les structures de gouvernance sans doute en raison, de la préparation de succession à la tête de ces entreprises (Welsch 1993). Les résultats obtenus par Belot et al. (2014) confirment la particularité des entreprises familiales, qui, dans le cadre de leur étude, ont tendance à adopter plus souvent une structure duale. Nos résultats confirment ce particularisme tout en soulignant que des changements plus fréquents de structure de gouvernance se produisent, en lien avec les phases de changement (succession, turnover) à la tête des entreprises familiales (Charlier et Lambert 2013). Ainsi, les entreprises familiales, souvent présentées comme des organisations très stables (de par leur actionnariat et la continuité du contrôle Missonier et Gundolf, 2017) affichent cependant une propension plus importante à faire évoluer leurs structures de gouvernance.

Nos résultats, en s'inspirant de l'intuition de Kole et Lehn $(1999,1997)$ et des pistes de recherche proposées notamment par Boivie et al. (2016) ou Wintoki et al. (2012) entendent apporter un éclairage inédit sur une dimension absente de la littérature sur la gouvernance. Ils montrent notamment le poids essentiel des principaux facteurs de gouvernance que l'on retrouve classiquement au cœur des relations d'agence (actionnaire, dirigeants, performance). Un examen global des changements de structure de gouvernance (comme dans le modèle 1) ne nous aurait donné qu'un aperçu superficiel. L'examen détaillé de l'influence des variables selon le type de transition permet ainsi d'analyser plus finement les processus sous-jacents. Par ailleurs, le double niveau de lecture proposé (par « modèle » et par « ligne ») permet ainsi d'approfondir les résultats obtenus dans le cadre de cet article. En ce sens, nos résultats ont une granularité plus fine et permettent d'examiner de façon précise les différents types de transition et les effets différenciés des variables.

Dans le cadre de cet article, nous n'avons pas totalement abordé la question de l'origine de l'instabilité de la structure de gouvernance. Quasiment absente de la littérature en gouvernance, le concept de stabilité (des systèmes) a largement été abordé dans de nombreux travaux en économie notamment. On peut considérer qu'un système génère, de manière endogène, sa propre instabilité comme on peut également considérer que l'instabilité d'un système provient de chocs exogènes entraînant des déséquilibres. En s'inspirant de cette dichotomie endogène/exogène, on peut considérer que la stabilité des structures de gouvernances pourrait être appréhendée par cette grille de lecture. En effet, la stabilité de la structure de gouvernance peut être affectée, de manière endogène, par l'évolution des relations entre les actionnaires, les administrateurs et les dirigeants. Les changements d'acteurs, d'orientations voire de rapport de forces pourraient in fine se traduire dans l'évolution de la structure de gouvernance (Bonnet et al. 2017). D'autre part, on peut considérer que l'évolution de structures de gouvernance peut résulter de chocs exogènes (évolution de l'industrie, évolution de la législation dont la soft law). Les changements de structures pourraient alors refléter l'adaptation aux évolutions de l'environnement $\mathrm{du}$ gouvernement de l'entreprise. Comme nous l'avons souligné précédemment ( $\mathrm{cf}$ note de bas de page $\mathrm{n}^{\circ} 15$ ), une approche de type " diff in diff» 
permettrait d'analyser l'effet d'une expérience naturelle sur les changements de la gouvernance des entreprises cotées françaises. On peut par exemple penser aux effets de la loi Copé/Zimmerman (2011) relative à la féminisation des conseils par exemple. Dans le cadre de cet article, nous avons essayé de traiter les différentes séries de facteurs susceptibles d'avoir une influence sur la stabilité de la structure de gouvernance. Nos modèles incluent aussi des facteurs de contingence que des variables capturant la dynamique interne de la gouvernance de l'entreprise ainsi qu'une variable identifiant l'année 2001 (loi NRE).

Nos résultats appellent plusieurs voies de recherche. Dans un premier temps, il nous paraît intéressant d'affiner la prise en compte du contexte dans lequel évolue l'entreprise. Nous venons de souligner l'intérêt potentiel de prendre en compte l'origine de l'instabilité potentielle de la structure de gouvernance. Une analyse documentaire approfondie permettrait de repérer les raisons avancées ou les justifications de l'évolution de la structure de gouvernance, que ce soit à travers la documentation fournie par l'entreprise comme la lettre aux actionnaires (Aerts et Yan 2017) ou le rapport annuel par exemple (Krause 2017). Une analyse documentaire des articles de presse permettrait également de mieux appréhender les transitions observées. Cela nous permettrait de repérer également les évènements les plus cruciaux dans le développement d'une entreprise telles que les opérations de fusion/acquisition qui entraînent de fait une évolution de la structure et de la gouvernance de l'entreprise fusionnée (Lel et Miller 2015). Par ailleurs, notre étude se focalise sur la stabilité de la structure de gouvernance en ne prenant pas en compte des éléments contextuels autour du dirigeant de l'entreprise. La littérature sur les dirigeants souligne que le contexte d'exercice du mandat du dirigeant n'est pas neutre (Occasio 1999). Un dirigeant interne ayant gravi peu à peu les échelons jusqu'au poste de DG est susceptible d'avoir entamé un processus d'enracinement quelques années auparavant. De façon plus globale, l'origine du DG (recrutement interne ou externe), sa réputation, son expérience et son réseau sont susceptibles de jouer sur l'exercice de son mandat et son maintien en poste (Zhang 2008). À ce titre, une piste de recherche intéressante semble se dessiner autour de l'impact potentiel des caractéristiques personnelles du DG. On peut penser ainsi à ajouter l'origine (externe/interne) du dirigeant ou encore la date d'entrée dans l'entreprise (Marcel et al. 2017). De la même manière, le contexte interne de gouvernance n'est pas neutre. À l'instar des dirigeants, les administrateurs peuvent être tentés de s'enraciner afin de conserver ou de gagner des mandats d'administrateur (Eminet et Guedri 2010). Ainsi, la dynamique interne et même interpersonnelle du conseil peut être étudiée (Westphal et Shani 2016) afin de saisir en quoi les administrateurs peuvent soutenir ou non le dirigeant en place dans sa volonté de maintenir ou faire évoluer la structure de gouvernance (Li, Krause, Qin, Zhang, Zhu, Lin, et Xu, 2018). De même, on ne peut pas écarter l'hypothèse de certains comportements mimétiques, qui en raison de la pression de l'environnement externe (évaluateurs, agences de notation, régulateurs) peuvent adopter le même type de structure afin de se conformer aux attentes des parties prenantes (Joseph et al 2014). Cela a par exemple été observé dans le secteur bancaire suite à la crise de 2008 (Grove et al. 2011). Enfin, le rôle des analystes financiers et des marchés financiers a été souligné de longue date et peut notamment pousser certaines entreprises à faire évoluer leur gouvernance afin de l'aligner avec les attentes des marchés voire de certains actionnaires (Aguilera, Desender, Bednar et Lee, 2015) Ces différents facteurs, qui peuvent être de nature fondamentalement endogène ou bien seulement des facteurs de contingence (Aguilera et al. 2008) sont susceptibles de favoriser ou non l'évolution potentielle de la 
structure de gouvernance et donc sa stabilité dans le temps. Ces quelques perspectives, si elles sont intégrées, pourront nous permettre à terme de mieux saisir les éléments les plus significatifs.

\section{Conclusion}

Notre article a pour objectif de s'interroger sur les variables susceptibles d'influencer la durée et la stabilité des structures de gouvernance des sociétés cotées françaises. Notre article entend ainsi examiner une dimension essentielle mais méconnue de la gouvernance. Pour cela, nous avons envisagé et testé une approche dynamique du choix et des transitions potentielles d'une forme de gouvernance à une autre. Nous intégrons ainsi l'influence du temps et des choix antérieurs dans l'analyse de l'évolution des formes de gouvernance des sociétés cotées françaises. En cela, nous contribuons tant du point de vue méthodologique (en mobilisant des modèles multi-états) que du point de vue empirique à une meilleure connaissance des facteurs affectant la stabilité et les transitions de structures de gouvernance. Nos résultats démontrent l'influence significative de certaines caractéristiques personnelles du dirigeant (notamment l'âge), de la performance et de la structure actionnariale des entreprises cotées. Nos résultats démontrent globalement une prime à la stabilité pour les plus grandes entreprises et les plus performantes. Un examen approfondi de nos variables indépendantes souligne l'influence contrastée de certaines variables en fonction du type de transition ou de gouvernance. Les pistes de recherches restent cependant nombreuses. Il pourrait être notamment intéressant d'intégrer dans des études postérieures les justifications avancées pour expliquer les changements de structures, mais également s'intéresser à l'impact potentiel du contexte d'enracinement du DG.

\section{BIBLIOGRAPHIE}

Aerts, W., et Yan, B., 2017, Rhetorical Impression Management In The Letter To Shareholders And Institutional Setting: A Metadiscourse Perspective, Accounting, Auditing \& Accountability Journal, vol. 30, n² 2, p. 404-432.

Aguilera, R., Desender, K., Bednar, M., et Lee, J., 2015, Connecting The Dots: Bringing External Corporate Governance Into The Corporate Governance Puzzle, The Academy of Management Annals, vol. 9, n 1, p. 483-573.

Aguilera, R. V., Filatotchev, I., Gospel, H. et Jackson, G., 2008, An Organizational Approach To Comparative Corporate Governance: Costs, Contingencies, And Complementarities, Organization Science, 19(3), p. 475-492.

Allemand I., et Brullebaut I., 2014, Le capital humain des femmes récemment nommées dans les conseils d'administration des sociétés françaises cotées à Paris, Management International, vol. $18, \mathrm{n}^{\circ} 3$, p. $20-31$ 
Allen, W., et Wood, G., 2006, Defining And Achieving Financial Stability, Journal of Financial Stability, Volume 2, $\mathrm{n}^{\circ}$ 2, p. 152-172.

Allison, P. D., (1982), Discrete-Time Methods for the Analysis of Event Histories, Sociological Methodology, vol. 13, p. 61-98.

Belot F., Ginglinger, E., Slovin, M. et M. Sushka 2014, Freedom Of Choice Between Unitary And Two-Tier Boards: An Empirical Analysis, Journal of Financial Economics, vol. 112 (issue 3), p. 364-385.

Boivie S., Bednar M., Aguilera R., et Andrus J., 2016, Are Boards Designed to Fail? The Implausibility of Effective Board Monitoring, Academy of Management Annals, vol. 10, $\mathrm{n}^{\circ} 1$, p. 319-407.

Bonnet, C., Seville, M., et Wirtz, P. (2017). Genèse et fonctionnement du conseil d'administration d'une firme entrepreneuriale : le rôle des identifications sociales des administrateurs, Finance Contrôle Stratégie, vol. 20, $\mathrm{n}^{\circ} 3$.

Boone A., Field Casares L., Karpoff J., et Raheja C., 2007, The Determinants Of Corporate Board Size And Composition: An Empirical Analysis, Journal of Financial Economics, vol. 85, p. 66-101.

Boyd, B. K., Gove S., et Solarino A., 2017, Methodological Rigor Of Corporate Governance Studies: A Review And Recommendations For Future Studies, Corporate Governance: An International Review, forthcoming.

Boyd, B. K., Haynes, K. T., et Zona, F., 2011, Dimensions of CEO-Board Relations, Journal of Management Studies, vol. 48, nº, p. 1892-1923.

Charlier P., et Lambert G., 2013, Modes de gouvernance et performances des entreprises familiales françaises en fonction des conflits d'agence, Finance Contrôle Stratégie, vol. 16, $\mathrm{n}^{\circ} 2$, p1-26.

Cleves, M. A., W. W. Gould, R. G. Gutierrez, et Y. V. Marchenko. 2010. An Introduction to Survival Analysis Using Stata. 3e ed. Stata Press.

Coleman James S. (1981). Longitudinal Data Analysis, Basic Books.

Conlon, A. S. C., Taylor, J. M. G., et Sargent, D. J. (2014). Multi-State Models For Colon Cancer Recurrence And Death With A Cured Fraction. Statistics in Medicine, 33(10), 1750-1766.

Crowther, M. J., 2016. MULTISTATE: Stata Module To Perform Multi-State Survival Analysis, Statistical Software Components, S458207, Boston College Department of Economics, revised 22 Nov 2016.

Crowther M. J., et Lambert P. C. (2014), A General Framework For Parametric Survival Analysis, Statistics in Medecine, 33, pages 5280-5297.

Daily C., Dalton, D., et Rajagopalan, N., 2003, Governance Through Ownership: Centuries of Practice, Decades of Research, Academy of Management Journal, vol. 46, p. 151-158.

Dalton, D. et Dalton, C., 2011, Integration of Micro and Macro Studies in Governance Research: CEO Duality, Board Composition, and Financial Performance, Journal of Management, vol. 37, $\mathrm{n}$ - 2, p. 404-411.

Drago, C., Millo, F., Ricciuti, R., et Santella, P., 2015, Corporate Governance Reforms, Interlocking Directorship And Company Performance In Italy, International Review of Law and Economics, vol. 41, p. 38-49. 
Eminet A., Guedri Z., 2010, The Role of Nominating Committees and Director Reputation in Shaping the Labor Market for Directors: An Empirical Assessment, Corporate Governance: an International Review, vol. 18, $\mathrm{n}^{\circ}$ 6, p. 557-574.

Eulenburg, C., Mahner, S., Woelber, L., et Wegscheider, K. (2015). A Systematic Model Specification Procedure for an Illness-Death Model without Recovery, PLoS ONE, vol. 10, $n^{\circ} 4$.

Faleye O., Mehrotra V., et Morck R., 2006, When Labor Has a Voice in Corporate Governance, Journal of Financial and Quantitative Analysis, vol. 41, n³, p. 489-510.

Fama E. et Jensen M., 1983, Separation of Ownership and Control, Journal of Law and Economics, vol. 26, p. 301-326.

Farewell, V. T., \& Tom, B. (2014). The Versatility Of Multi-State Models For The Analysis Of Longitudinal Data With Unobservable Features, Lifetime Data Analysis, vol. 20, n 1, p. 51-75.

Filatotchev, I., Toms, S., et Wright, M. 2006. The Firm's Strategic Dynamics and Corporate Governance Life-Cycle, International Journal of Managerial Finance, vol. 2, n 4, p. 256-279.

Fracassi, C., et Tate, G., 2012, External Networking and Internal Firm Governance, Journal of Finance, vol. 67, $\mathrm{n}^{\circ}$ 1, p. 153-194.

Francis, B., Hasan, I., et Wu, Q., 2015, Professors in The Boardroom And Their Impact on Corporate Governance and Firm Performance, Financial Management, vol. 44, n 3, p. 547-581.

Foucher Y., Mathieu E., Saint-Pierre P., Durand J. et Daurès J.-P., 2004, A Semi-Markov Model Based On Generalized Weibull Distribution With An Illustration For Hiv Disease, Biometrical Journal, vol. 47, n 6, p. 825-833.

Gauzère, F., Commenges, D., Barberger-Gateau, P., Letenneur, L. et Dartigues, J.-F. (1999). Maladie et dépendance - Description des évolutions par des modèles multiétats. Population, vol. $52, \mathrm{n}^{\circ} 4$, p. 205-222.

Goyal V. et Park C., 2002, Board Leadership Structure And CEO Turnover, Journal of Corporate Finance, vol. 8, $\mathrm{n}^{\circ}$ 1, p. 49-66.

Gregoir S., Maury T-P., et Palomino F., 2013, La féminisation des Conseils d'Administration des grandes entreprises en France : au-delà des apparences, document de travail EDHEC. http:// docs.edhec-risk.com/rsc/130715/EDHEC_PP_Feminisation_CA.pdf

Grove, H., Patelli, L., Victoravich, L. M., et Xu, P. 2011 Corporate Governance and Performance in the Wake of the Financial Crisis: Evidence from US Commercial Bank, Corporate Governance: An International Review, vol. 19, n5, p. 418-436.

Hermalin B., et Weisbach M., 2003, Board Of Directors As An Endogenously Determined Institution: A Survey Of Economic Literature, FRBNY Economic Policy Review, Avril, p. 7-26.

Hollandts X., Borodak D., et Tichit A., 2015, Plus autonome ? L'évolution des structures de gouvernance des sociétés cotées françaises (1997-2008), Management International, vol. 19, nº 3, p. 47-64.

Huber-Carol C. et Vonta I. (2004). Frailty Models For Arbitrarily Censored And Truncated Data, Lifetime Data Analysis, vol. 10, p. 369-388.

Jackson, C., 2014, Multi-State Modelling Software, And Encouraging Statistical Software Development. MRC Biostatistics Unit, Cambridge, U.K., MRC Biostatistics Unit Centenary Conference, 25 March 2014.

Jarrow, R. A. et Turnbull, S. (1995). Pricing Derivatives on Financial Securities Subject to Credit Risk, Journal of Finance, vol. 50, n 1, p. 53-85. 
Johnson P. E., 2014, Formal Theories of Politics: Mathematical Modelling in Political Science. Elsevier.

Joseph, J., Ocasio, W., et McDonnell, M.-H., 2014, The Structural Elaboration of Board Independence: Executive Power, Institutional Logics, and the Adoption of CEO-Only Board Structures in U.S, Corporate Governance. Academy of Management Journal, vol. 57, $\mathrm{n}^{\circ} 6,1834-$ 1858.

Kole S. R., et Lehn K. M., 1997, Deregulation, the Evolution of Corporate Governance Structure and Survival, American Economic Review, vol. 87, n 2, p. 421-425

Kole, S. R., et Lehn, K. M., 1999, Deregulation And The Adaptation Of Governance Structure: The Case Of The U.S. Airline Industry, Journal of Financial Economics, vol. 52, n 1, p. 79-117.

Koopman, S. J., Lucas, A. et Monteiro, A. (2008). The Multi-State Latent Factor Intensity Model For Credit Rating Transitions, Journal of Econometrics, vol. 142, n 1, p. 399-424.

Krause, R. 2017. Being The CEO's Boss: An Examination of Board Chair Orientations, Strategic Management Journal, vol. 38, n 3, p. 697-713.

Lel, U., et Miller, D., 2015, Does Takeover Activity Cause Managerial Discipline? Evidence From International M\&A Laws, Review of Financial Studies, vol. 28, n 6, p. 1588-1622.

Li, W., Krause, R., Qin, X., Zhang, J., Zhu, H., Lin, S., et Xu, Y., 2018, Under The Microscope: An Experimental Look At Board Transparency And Director Monitoring Behavior, Strategic Management Journal, forthcoming.

Linck, J., Netter J., et Yang T., 2008, Determinants of Board Structure, Journal of Financial Economics, vol. 87, p. 308-328.

Marcel, J., Cowen, A., et Ballinger, G., 2017, Are Disruptive CEO Successions Viewed as a Governance Lapse? Evidence From Board Turnover, Journal of Management, vol. 43, n 5, p. 13131334.

Mard, Y., Marsat, S., et Roux, F., 2014, Structure De L'actionnariat et Performance Financière De L'entreprise : Le Cas Français. Finance Contrôle Stratégie, vol. 17, n 4, p. 59-83.

Missonier A. et Gundolf, K., 2017, L'entreprise familiale : état et perspectives de la recherche francophone, Finance Contrôle Stratégie, vol. 20, n² 2, p. 1-32.

Occasio W., 1999, Institutionalized Action and Corporate Governance: The Reliance on Rules of CEO Succession, Administrative Science Quaterly, vol. 44, $\mathrm{n}^{\circ}$ 2, p. 384-416.

Perez-Ocon R. et Ruiz-Castro J. E. (1999). Semi-Markov Models And Applications, chapter 14, p. 229-238. Kluwer Academic Publishers.

Raheja C., 2005, Determinants of Board Size and Composition: A Theory of Corporate Boards, Journal of Financial and Quantitative Analysis, vol. 40, nº 2, p. 283-306.

Shleifer A. et Vishny R., 1997, A Survey Of Corporate Governance, Journal of Finance, vol. 52, p. $737-783$.

Sur, S., Lvina, E., et Magnan, M. 2013. Why do Boards Differ? Because Owners Do: Assessing Ownership Impact on Board Composition, Corporate Governance: An International Review. vol. $21, n^{\circ} 4$, p. 373-389

Tihanyi L., Graffin S., et George G., 2014, Rethinking Governance in Management Research: From the Editors, Academy of Management Journal. vol. 57, n 6, p. 1535-1543. 
Welsch, J., 1993, The Impact of Family Ownership and Involvement on the Process of Management Succession, Family Business Review, vol. 6, $\mathrm{n}^{\circ}$ 1, p. 31-54.

Westphal J., 1998, Board Games: How CEOs Adapt to Increases in Structural Board Independence from Management, Administrative Science Quarterly, vol. 43, n 3, p. 511-537.

Westphal, J. D., et Shani, G., 2016, Psyched-Up To Suck-Up: Self-Regulated Cognition, Interpersonal Influence, And Recommendations For Board Appointments in The Corporate Elite, Academy of Management Journal, vol. 59, n², p. 479-509.

Wiberg, M., Marklund, S., et Alexanderson, K. (2017). Transitions Between Compensated Work Disability, Joblessness, and Self-Sufficiency: A Cohort Study 1997-2010 of Those Jobless in 1995, Population Research and Policy Review, vol. 36, $\mathrm{n}^{\circ}$ 1, p. 85-107.

Wintoki, M. B., Linck, J. S., et Netter, J. M., 2012, Endogeneity and The Dynamics Of Internal Corporate Governance, Journal of Financial Economics, vol. 105, nº 3, p. 581-606.

Wirtz P., 2008, Les meilleures pratiques de gouvernance d'entreprise, La Decouverte.

Yamaguchi K. (1991). Event History Analysis. Sage Publications.

Zajac E. et Westphal P., 1995, Who Shall Govern? CEO/Board Power, Demographic Similarity, And New Director Selection, Administrative Science Quarterly, n 40, p. 60-83.

Zhang Y., 2008, Information Asymmetry and Dismissal of Newly Appointed CEOs, Strategic Management Journal, vol. 29, p. 859-872.

\section{ANNEXES}

Annexe 1 : Tableau Statistiques descriptives selon le type de transition potentielle

\begin{tabular}{|c|c|c|c|c|c|c|c|c|c|}
\hline & $\begin{array}{l}\text { Transition } \\
1 \& 2\end{array}$ & $\begin{array}{l}\text { Transitions } \\
3 \& 4 \& 5 \& 6\end{array}$ & & $\begin{array}{l}\text { Transition } \\
3 \& 4 \S\end{array}$ & $\begin{array}{l}\text { Transitions } \\
1 \& 2 \& 5 \& 6\end{array}$ & & $\begin{array}{l}\text { Transition } \\
5 \& 6\end{array}$ & $\begin{array}{l}\text { Transitions } \\
1 \& 2 \& 3 \& 4\end{array}$ & \\
\hline & $\begin{array}{l}F G 1->F G 2 \\
F G 1 \text {->FG3 }\end{array}$ & & & $\begin{array}{l}F G 2->F G 1 \\
F G 2->F G 3\end{array}$ & & & $\begin{array}{l}\text { FG3->FG1 } \\
\text { FG3->FG2 }\end{array}$ & & \\
\hline & $\begin{array}{l}\text { mean } \\
\text { value } \\
\text { mu_1 }\end{array}$ & $\begin{array}{l}\text { mean } \\
\text { value } \\
\text { mu_2 }\end{array}$ & $\begin{array}{l}\text { Difference } \\
(t \text {-value }) d / \\
\text { d_se }\end{array}$ & $\begin{array}{l}\text { mean } \\
\text { value } \\
\text { mu_1 }\end{array}$ & $\begin{array}{l}\text { mean } \\
\text { value } \\
\text { mu_2 }\end{array}$ & $\begin{array}{l}\text { Difference } \\
\text { (t-value) } \\
\text { d/d_se }\end{array}$ & $\begin{array}{l}\text { mean } \\
\text { value } \\
\text { mu_1 }\end{array}$ & $\begin{array}{l}\text { mean } \\
\text { value } \\
\text { mu_2 }\end{array}$ & $\begin{array}{l}\text { Difference } \\
\text { (t-value) } \\
\text { d/d_se }\end{array}$ \\
\hline \multirow[t]{2}{*}{ boucle } & 0,20 & 0,31 & $-0,12^{* * *}$ & 0,26 & 0,36 & $-0,10^{* * *}$ & 0,32 & 0,09 & $0,23 * * *$ \\
\hline & & & $(0,02)$ & & & $(0,03)$ & & & $(0,02)$ \\
\hline \multirow[t]{2}{*}{ performance } & 8,82 & 9,22 & $-0,40$ & 9,44 & 4,68 & 4,77 & 8,19 & 11,46 & $-3,27$ \\
\hline & & & $(1,84)$ & & & $(3,29)$ & & & $(2,07)$ \\
\hline $\begin{array}{l}\text { Concentration } \\
\text { actionnariat }\end{array}$ & 35,84 & 37,15 & $-1,31$ & 36,72 & 35,21 & 1,50 & 36,71 & 36,25 & 0,46 \\
\hline
\end{tabular}




\begin{tabular}{|c|c|c|c|c|c|c|c|c|c|}
\hline & & & $(0,84)$ & & & $(1,50)$ & & & $(0,94)$ \\
\hline \multirow{2}{*}{$\begin{array}{l}\% \text { _femmes au } \\
\text { conseil }\end{array}$} & 13,50 & 13,28 & 0,22 & 13,32 & 13,97 & $-0,65$ & 13,44 & 13,20 & 0,24 \\
\hline & & & $(0,47)$ & & & $(0,84)$ & & & $(0,53)$ \\
\hline \multirow[t]{2}{*}{ Age_dirigeant } & 53,90 & 56,35 & $-2,44^{* * *}$ & 55,38 & 54,45 & 0,93 & 55,92 & 53,56 & $2,36^{* * *}$ \\
\hline & & & $(0,27)$ & & & $(0,49)$ & & & $(0,31)$ \\
\hline \multirow[t]{2}{*}{$\begin{array}{l}\text { Actif } \\
\text { intangible }\end{array}$} & $3,29 e+06$ & $4,10 \mathrm{e}+06$ & $-8,11 e+05^{* *}$ & $3,67 e+06$ & $4,68 \mathrm{e}+06$ & $-1,01 e+06$ & $4,23 e+06$ & $2,40 e+06$ & $\begin{array}{l}1,83 e \\
+06^{* * *}\end{array}$ \\
\hline & & & $(305917,68)$ & & & $(547201,86)$ & & & $(343066,48)$ \\
\hline \multirow[t]{2}{*}{ endettement } & 38,82 & 40,73 & $-1,91^{*}$ & 39,87 & 40,42 & $-0,56$ & 40,66 & 37,80 & $2,86^{* * *}$ \\
\hline & & & $(0,77)$ & & & $(1,38)$ & & & $(0,87)$ \\
\hline \multirow[t]{2}{*}{ Total actif } & 11907,21 & 17415,72 & $-5508,51^{* * *}$ & 15055,27 & 14992,69 & 62,58 & 16866,17 & 9937,06 & $6929,10^{* * *}$ \\
\hline & & & $(1111,07)$ & & & $(1994,59)$ & & & $(1249,26)$ \\
\hline \multirow[t]{2}{*}{$\begin{array}{l}\text { Chiffre } \\
\text { d'affaires }\end{array}$} & 8264,76 & 11704,93 & $-3440,17^{* * *}$ & 10233,95 & 10157,07 & 76,88 & 11353,87 & 7056,48 & $4297,39 * * *$ \\
\hline & & & $(717,19)$ & & & $(1287,15)$ & & & $(806,50)$ \\
\hline
\end{tabular}

Note: La transition 4 de FG2 vers FG3 n'a pas d'occurrence dans notre échantillon.

Annexe 2 : l'évolution de la structure de gouvernance pour l'ensemble des entreprises 


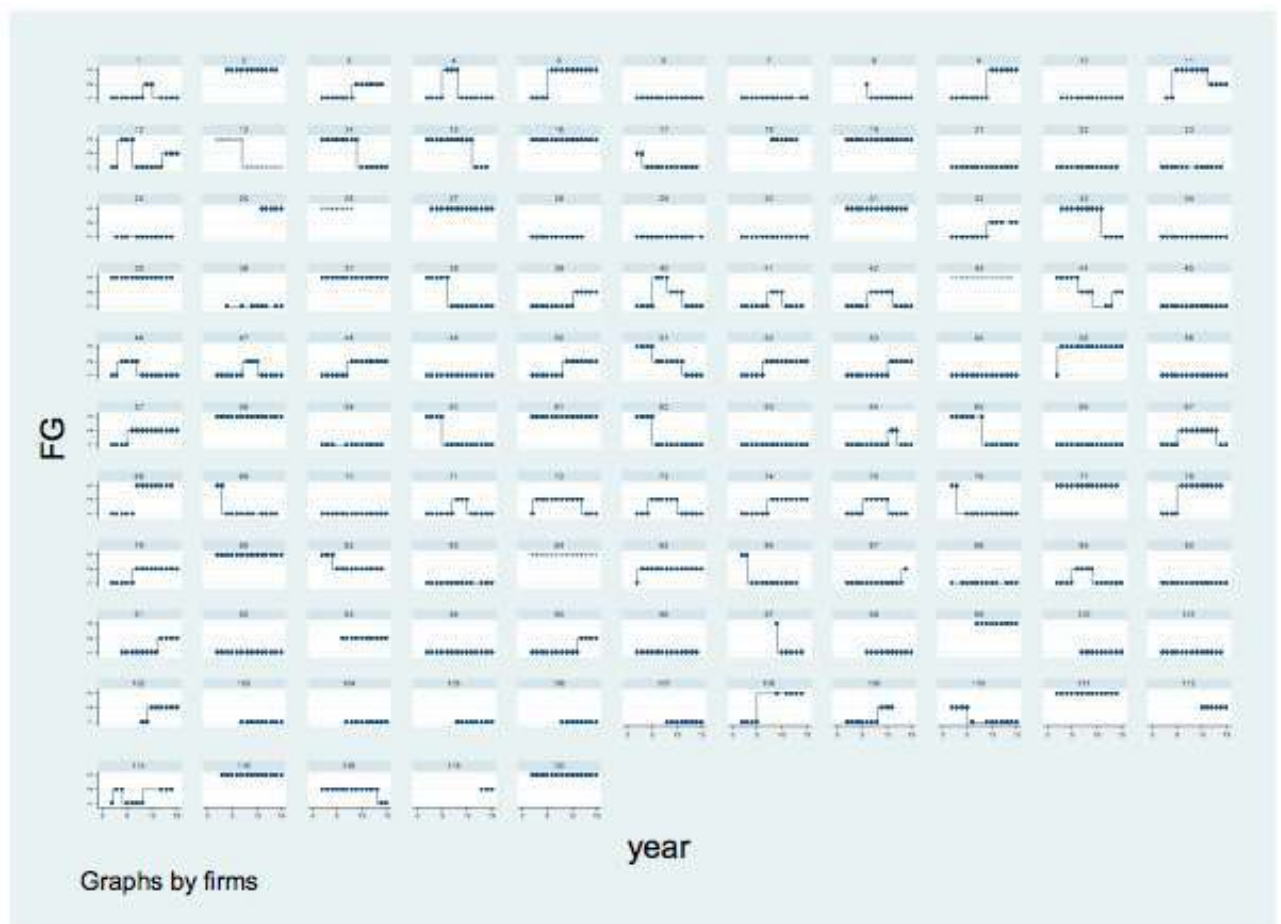

Annexe 3 : Présentation détaillée du schéma de Markov utilisé pour analyser la dynamique de changement de la structure de gouvernance.

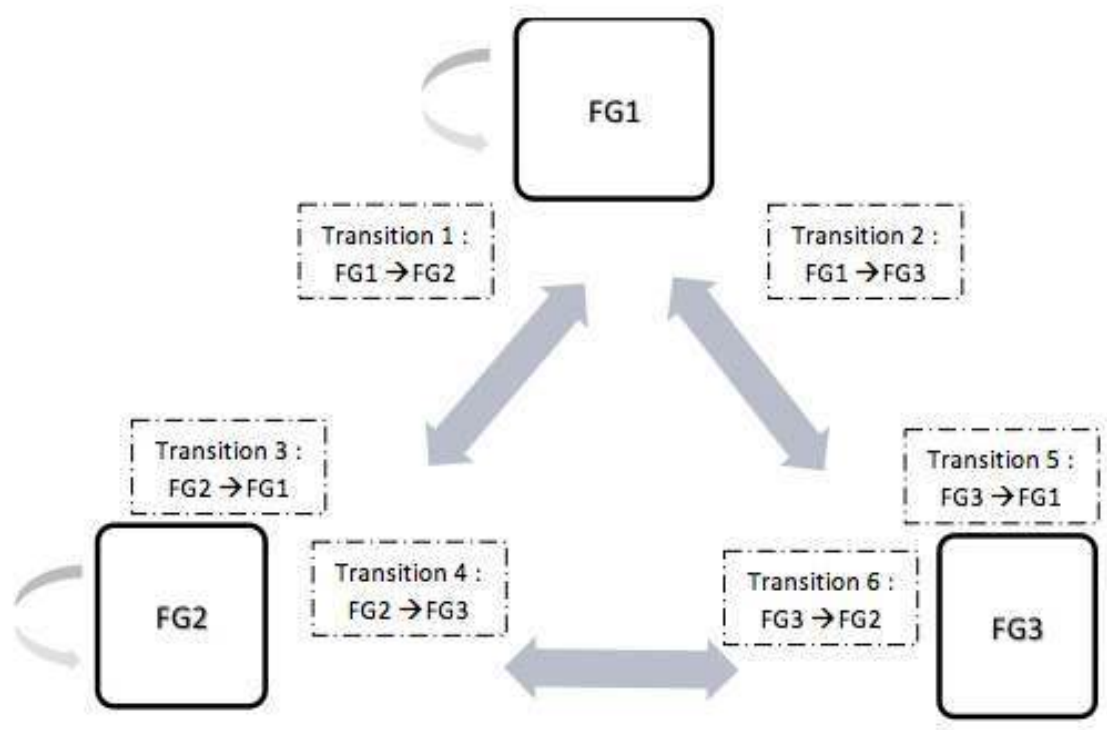


Nous considérons $Y(t)$ le processus d'évolution, dans le temps, de la forme de gouvernance (FG) d'une entreprise. Dans l'ensemble de la population statistique, le processus $\{Y(t), t \geq 0\}$ est une variable aléatoire c'est-à-dire que le moment d'occurrence de l'évènement (transition d'un état à un autre) varie selon les individus statistiques, ici les entreprises, qui prend des valeurs dans un espace fini de 3 états $S=\{1, \ldots, 3\}$. L'évènement, ici la variation de $Y t$ est noté $\Delta Y t$ et peut prendre la valeur 1 ou 0 , correspondant respectivement au fait que l'évènement s'est réalisé ou non.

Notons $T$ la variable aléatoire qui représente la durée observée dans un état initial avant la transition vers un autre état. Notre intérêt porte sur la distribution de la probabilité de connaître l'évènement durant l'intervalle de temps $\left[t, t^{\prime}\right]$, sachant que les entreprises ne l'ont pas encore connu à l'instant $\mathrm{t}$ :

$$
R\left(\Delta Y_{t}=1\right)=P\left(t \leq T<t^{\prime} / T \geq t\right)
$$

Etant donné que nous disposons de mesure de durées d'occurrence d'évènements sur la base d'une unité de temps longue, l'année, nous considérons ici une approche en temps discret. La probabilité de connaître un évènement au temps $t_{\iota}$ sachant que l'on n'avait pas encore connu cet évènement auparavant est :

$$
P\left(t_{i}\right)=P\left(T t_{l} / t T \geq t\right)
$$

Deux types de facteurs sont considérés dans l'analyse des différences individuelles dans les rythmes de transition d'un état à un autre: facteurs liés à l'environnement et des caractéristiques des entreprises (invariante au cours du temps ou dépendantes du temps). Cet ensemble de $\mathrm{k}$ variables, noté $x_{t}$ est définit ainsi :

$$
x_{a}\left(x_{l b}, x_{2 \text { tansess }} x_{k i}\right) \quad \text { avec } j=(1,2, \ldots, k)
$$

Le risque de connaître un évènement s'écrit alors :

$$
h(t)=f\left(t, x_{t}\right)
$$

avec $\mathrm{h}(\mathrm{tj})$ le risque qui est fonction du temps et des caractéristiques xi(Yamaguchi, 1991).

La formulation de l'équation (4) correspond aux modèles de durée avec hypothèse de dépendance temporelle à des données à risques concurrents (distributions supposant que la probabilité de sortie d'un état dépend du temps passé jusque-là dans l'état) et revient à dire que la probabilité de chaque évènement dépend non seulement de la forme de gouvernance observée à cet instant, mais également du temps passé jusque-là. Ainsi, les probabilités de transition dépendent intrinsèquement du temps passé et de la forme observée à l'état critique dès lors qu'un modèle de durée avec dépendance temporelle est appliqué (Bijwaard, 2014). Par conséquent, pour estimer l'équation (4) nous adoptons ici l'approche paramétrique qui consiste à formuler des hypothèses sur la forme de distribution du risque $\mathrm{h}(\mathrm{ti})$ au cours du

temps. Enfin, nous considérons que le modèle peut également faire intervenir un effet aléatoire pour tenir compte de la dépendance entre les temps d'évènement ou de l'omission d'une ou plusieurs caractéristiques des individus statistiques dans le modèle-appelés modèle de fragilité ( frailty model $»^{17}$ — Heckman et Singer, 1984).

\section{Annexe 4 : choix du modèle}

Pour chacune des six lois de distribution utilisées, le tableau ci-dessous résume les transitions qui semblent vérifier l'hypothèse de risques proportionnels. Le symbole $\times$ signifie que la transition ne vérifie pas l'hypothèse et le symbole $O$ signifie que la transition vérifie l'hypothèse.

L'ensemble des résultats sont disponibles sur demande. 


\begin{tabular}{|c|c|c|c|c|c|c|}
\hline & $\begin{array}{l}\text { Modèle } 1 \\
\text { Distrib. } \\
\text { Exp. Avec } \\
\text { frailty }\end{array}$ & $\begin{array}{l}\text { Modèle } 2 \\
\text { Distrib. } \\
\text { Gompertz }\end{array}$ & $\begin{array}{l}\text { Modèle } \\
\text { loglogistic }\end{array}$ & $\begin{array}{l}\text { Modèle } 4 \\
\text { Distrib. } \\
\text { Weibull }\end{array}$ & $\begin{array}{l}\text { Modèle } 5 \\
\text { lognormal }\end{array}$ & $\begin{array}{l}\text { Modèle } 6 \\
\text { Distrib. } \\
\text { Ggamma }\end{array}$ \\
\hline $\begin{array}{l}\text { ech total et } \\
\text { ensemble des } \\
\text { variables }\end{array}$ & 0 & 0 & $\mathrm{X}$ & $\mathrm{X}$ & $\mathrm{X}$ & $\mathrm{X}$ \\
\hline $\begin{array}{l}\text { Ech. Total et } \\
\text { sélection des } \\
\text { variables }\end{array}$ & 0 & 0 & $\mathrm{X}$ & 0 & $\mathrm{X}$ & $\mathrm{X}$ \\
\hline $\begin{array}{l}\text { Sous-éch. } \\
\text { Transition1\&2 }\end{array}$ & 0 & 0 & 0 & 0 & $x$ & $\mathrm{x}$ \\
\hline $\begin{array}{l}\text { Sous-éch. } \\
\text { Transition1 }\end{array}$ & 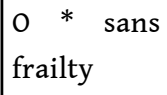 & 0 & 0 & 0 & $\mathrm{X}$ & $\mathrm{X}$ \\
\hline $\begin{array}{l}\text { Sous-éch. } \\
\text { Transition2 }\end{array}$ & 0 & $\mathrm{X}$ & $\mathrm{X}$ & $\mathrm{X}$ & $\mathrm{X}$ & $\mathrm{X}$ \\
\hline $\begin{array}{l}\text { Sous-éch. } \\
\text { Transition3 }\end{array}$ & $X$ & $\mathrm{X}$ & 0 & 0 & $\mathrm{X}$ & $\mathrm{X}$ \\
\hline $\begin{array}{l}\text { Sous-éch. } \\
\text { Transition 5\&6 }\end{array}$ & 0 & 0 & 0 & 0 & $\mathrm{X}$ & $\mathrm{X}$ \\
\hline $\begin{array}{l}\text { Sous-éch. } \\
\text { Transition5 }\end{array}$ & 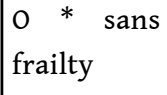 & $\mathrm{X}$ & 0 & 0 & $\mathrm{X}$ & $\mathrm{X}$ \\
\hline $\begin{array}{l}\text { Sous-éch. } \\
\text { Transition6 }\end{array}$ & $\begin{array}{l}0 * \text { sans } \\
\text { frailty }\end{array}$ & $\mathrm{X}$ & $\mathrm{X}$ & $\mathrm{X}$ & $\mathrm{X}$ & $\mathrm{X}$ \\
\hline
\end{tabular}

Annexe Tableau 3 suite : Résultats des régressions des modèles multi-états testant les effets des variables de gouvernance et de performance sur la durée et le type de transition observée

\begin{tabular}{|l|l|l|}
\hline Modèle & 7 & 8 \\
\hline & (Sous-échantillon) & (Sous-échantillon) \\
\hline & Transition1\&2 & Transition 5\&6 \\
\hline Type de distribution & FG1 à FG2 et FG1 à FG3 & FG3 àFG1 et FG3 àFG2 \\
\hline & Distrib. Exp. Avec frailty & Distrib. Gompertz \\
\hline & Likelihood ratio (Proba) & Likelihood ratio (Proba) \\
\hline
\end{tabular}




\begin{tabular}{|c|c|c|}
\hline boucle & $8,79 *(0,09)$ & $10,91^{* * *}(0,00)$ \\
\hline Année 2001 & $3,52(0,42)$ & $0,00^{* * *}(0,00)$ \\
\hline Performance & $1,03^{* *}(0,04)$ & $0,97(0,63)$ \\
\hline CA/actions & $0,99 * *(0,03)$ & $1,03 *(0,07)$ \\
\hline Concentration actionnariat & $0,99(0,35)$ & $1,00(0,95)$ \\
\hline Actionnariat salarié & $0,91(0,25)$ & $1,10^{* *}(0,03)$ \\
\hline État & $1,34(0,66)$ & $2,02(0,41)$ \\
\hline Entreprise & $0,45(0,48)$ & $2,14(0,37)$ \\
\hline Famille & $0,35(0,28)$ & $0,89(0,89)$ \\
\hline$\%$ femmes au conseil & $0,99(0,61)$ & $0,99(0,68)$ \\
\hline Âge dirigeant & $1,22^{* * *}(0,01)$ & $1,01(0,64)$ \\
\hline Actifs intangibles & $1,00(0,20)$ & $1,00^{* * *}(0,01)$ \\
\hline Endettement & $1,01(0,31)$ & $1,00(0,84)$ \\
\hline Chiffre d'affaires & $1,00(0,22)$ & $1,00 *(0,06)$ \\
\hline Productivité & $1,00(0,18)$ & $1,00(0,40)$ \\
\hline Constant & $0,00^{* * *}(0,00)$ & $0,00(0,16)$ \\
\hline Secteurs (SIC code) & Oui & Oui \\
\hline Observations & 1636 & 758 \\
\hline $\mathrm{Nb}$ d'entreprises & 91 & 45 \\
\hline chi2 & $35,92^{* * *}$ & $2192,46^{* * *}$ \\
\hline aic & $-125,93$ & $-54,05$ \\
\hline bic & $-17,74$ & 38,87 \\
\hline
\end{tabular}

Note: La transition 4 de FG2 vers FG3 n'a pas d'occurrence dans notre échantillon.

\section{NOTES}

1. Les entreprises françaises ont la faculté de pouvoir opter pour trois modalités et deux types de structures de conseil : la première structure est représentée par le conseil d'administration avec ou sans séparation des fonctions de Président/Directeur Général (première et seconde modalité) 
et une seconde structure, la forme duale (directoire et conseil de surveillance - troisième modalité).

2. De façon générale, la stabilité d'un système est définie par la négative c'est-à-dire son absence d'instabilité. Ainsi, Allen et Wood (2006: 152) définissent la stabilité financière d'une entreprise " as a state of affairs in which episodes of instability are unlikely to occur».

3. Les modèles multi-états permettent l'analyse de la durée dans un " état » et la " transition » d'un état à un autre, marqué par un " évènement » qui délimite dans le temps le passage d'une situation d'origine à une situation d'arrivée (Coleman, 1981). Ces modèles permettent également de prendre en compte des allers-retours entre plusieurs états (voir la section 2 pour plus de détails).

4. L'absence d'études mobilisant des modèles de durée peut à la fois s'expliquer par la complexité statistique de la modélisation, mais également par l'absence jusqu'ici de logiciels permettant de réaliser de telles estimations de façon aisée (Jackson 2014).

5. Les "transitions" correspondent dans le cas présent au passage à une autre forme de gouvernance parmi les trois formes possibles.

6. Pour la description détaillée de cet échantillon, se référer à la section 2.2.

7. https://investir.lesechos.fr/actions/actualites/ag-maurice-levy-va-transmettre-les-renes-dugroupe-au-roi-arthur-1681134.php

8. Dans notre cas la censure des données signifie que nous observons les entreprises dans un intervalle donné et nous ne disposons pas d'informations sur les évènements en amont et en aval de cet intervalle. Nous sommes en présence de censure indépendante de l'occurrence des évènements ; ici les entreprises n'entrent pas ou ne sortent pas de l'échantillon en fonction du changement de leur forme de gouvernance.

9. C'est le paramètre $\Delta Y t$ dans nos modèles d'estimation, qui peut prendre la valeur 1 ou 0 , correspondant respectivement au fait que l'évènement s'est réalisé ou non.

10. Comme c'est le cas par exemple dans le cas des entreprises 10 et 114 dans le graphique 1 cidessus.

11. Les modèles de risques concurrents (ou épisodes multiples) les plus utilisés supposent une chaîne de dépendance entre les évènements de type Markov. Une chaîne de Markov suppose ici que la probabilité de transition dépend de la forme de gouvernance dans laquelle on se trouve à la période considérée.

12. Pour deux des 120 entreprises, certaines données sont manquantes ce qui nous a conduits à les exclure de notre échantillon final.

13. Nous avons testé trois variables concernant le dirigeant: son âge, la durée dans le poste et le turnover du dirigeant. Du point de vue statistique, ces trois variables sont en général relativement corrélées entre elles. Les différentes variables ont été introduites de façon séparée puis ensemble dans les différentes estimations. A chaque fois, les variables relatives à la durée en poste et le turnover n'ont pas été retenues par le logiciel, bien souvent pour des raisons de multicolinéarité entre les variables.

14. La probabilité de chaque évènement dépend non seulement de la forme de gouvernance observée à cet instant, mais également du temps passé jusque-là. Nous adoptons ici l'approche paramétrique qui consiste à formuler une hypothèse sur la forme de distribution du risque $h(t j)$ au cours du temps. Nous avons ainsi retenu la distribution Gamma généralisée (Ggamma), au regard des critères AIC et BIC, comme hypothèse sur la distribution du risque dans le cas de l'évolution des formes de gouvernance des sociétés françaises.

15. A ce titre, une méthodologie alternative telle que la méthode difference-in-difference peut être mobilisée pour analyser les effets d'un changement ou d'évolutions du cadre réglementaire ou législatif de la gouvernance des sociétés françaises. Classiquement mobilisée dans l'évaluation des politiques publiques (fiscalité, emploi par exemple), elle est de plus en plus utilisée dans la recherche en gouvernance (Francis, Hasan et Wu, 2015). En général, cela suppose l'existence 
d'une « expérience naturelle » telle que l'application d'un nouveau code de gouvernance ou un changement législatif (Drago et al. 2015).

\section{RÉSUMÉS}

Cet article se donne pour objectif d'examiner une dimension cruciale et pourtant méconnue de la gouvernance des entreprises. Nous étudions la dynamique de la gouvernance des sociétés cotées françaises (SBF 120) sur une longue période d'observation (2000-2014) qui englobe les principales réformes françaises. Plus précisément, nous analysons les variables susceptibles d'influencer la stabilité de la structure de gouvernance. Pour cela, nous mobilisons des modèles de durée multiétats de type semi-markovien afin d'analyser à la fois la durée et les transitions d'une forme de gouvernance à une autre des sociétés cotées françaises. Nos résultats montrent que la stabilité de la structure de gouvernance est essentiellement liée aux caractéristiques personnelles du dirigeant en poste, à la performance de l'entreprise et la structure actionnariale. Cependant, un examen approfondi par type de transition montre l'influence multiple et parfois contrastée de certaines variables.

The aim of this article is to examine a crucial and yet unknown aspect of corporate governance. More precisely, we analyze the variables which are likely to influence the stability of the governance structure of French listed companies (SBF 120 index). We study the dynamics of corporate governance over a long period of observation (2000-2014), which encompasses the main French reforms. We use duration models based on Markovian-type processes to examine both the transitions from one form of governance to the other, but also the duration and therefore the stability of the forms of governance of listed French companies. Our results show that the stability of the corporate governance structure is linked to the personal characteristics of the CEO, the performance of the company and the shareholder structure essentially. However, a thorough examination by type of transition shows the multiple and sometimes contrasted influence of some variables. Our article brings a new light on the dynamics of the governance of French listed companies.

\section{INDEX}

Keywords : corporate governance, board of directors, Markov multi-state models.

Mots-clés : gouvernement d'entreprise - conseil d'administration - conseil de surveillance directoire - modèle de durée - chaînes de Markov - modèle multi-états

jel G30 - General, J54 - Producer Cooperatives; Labor Managed Firms, M54 - Labor Management (team formation; worker empowerment; job design; tasks and authority; job satisfaction)

\section{AUTEURS}

\section{XAVIER HOLLANDTS}

KEDGE \& CRCGM 
DANIELA BORODAK

ESC Clermont, CRCGM \& CERDI

ARIANE TICHIT

UCA \& CERDI 